\title{
Behavioral expression of cocaine sensitization in rats is accompanied by a distinct pattern of modifications in the PKA/DARPP-32 signaling pathway
}

\author{
Simona Scheggi, Anna Raone, Maria Graziella De Montis, Alessandro Tagliamonte and \\ Carla Gambarana
}

Department of Neuroscience, Pharmacology Unit, University of Siena, Siena, Italy

\begin{abstract}
Repeated cocaine administration induces behavioral sensitization and modifications in the phosphorylation pattern of dopamine and cAMP-regulated phosphoprotein of $\mathrm{M}_{\mathrm{r}} 32000$ (DARPP-32), characterized by a tonic increase in the Thr75 phosphorylated form, and a decrease in the Thr34 phosphorylated form. This study further investigated the correlations between cocaine sensitization and modifications in the DARPP-32 phosphorylation pattern, cAMP-dependent protein kinase (PKA) activity, and mGluR5 tone in the medial prefrontal cortex and nucleus accumbens. Behavioral sensitization and modifications in these neurochemical markers followed a similar temporal pattern. Moreover, in sensitized rats acute cocaine administration modified phosphorylation levels of Thr75- and Thr34-DARPP-32, GluR 1 , and NR 1 subunits in the nucleus accumbens only at a dose double the
\end{abstract}

efficacious dose in control rats. These results suggest that the high levels of phospho-Thr75 DARPP-32 maintain PKA in a prevalent inhibited state. Furthermore, in sensitized rats the acute administration of 6-methyl-2-(phenylethynyl)-pyridine, a mGluR5 antagonist, reinstated the phosphorylation levels of Thr75- and Thr34-DARPP-32, GluR 1 , and $\mathrm{NR}_{1}$ to control values, and a subsequent cocaine challenge did not elicit a sensitized response. These data suggest that a tonic increase in mGluR5 transmission in cocaine-sensitized rats sustains both the increase in phospho-Thr75 DARPP-32 levels and the expression of behavioral sensitization.

Keywords: 6-methyl-2-(phenylethynyl)-pyridine, dopamine and cAMP-regulated phosphoprotein of $M_{r} 32000$, medial prefrontal cortex, nucleus accumbens.

J. Neurochem. (2007) 103, 1168-1183.
The dopamine and cAMP-regulated phosphoprotein of $\mathrm{M}_{\mathrm{r}}$ 32000 (DARPP-32) (Walaas et al. 1983) is enriched in medium spiny neurons in dorsal and ventral striatum (Ouimet et al. 1984), where it plays a central role in integrating dopaminergic and glutamatergic neurotransmission originating mainly from cortical areas and the substantia nigra pars compacta/ventral tegmental area, respectively (Greengard 2001). DARPP-32 is a major target protein for the cAMPdependent protein kinase (PKA) and its function is determined by the phosphorylation state. Dopamine, by means of the $\mathrm{D}_{1}$ receptor-mediated activation of PKA, phosphorylates DARPP-32 at threonine-34 (Thr-34) and thereby converts DARPP-32 into a potent inhibitor of protein phosphatase-1 (Hemmings et al. 1984). Phosphorylation at threonine-75 (Thr-75) converts DARPP-32 into an inhibitor of PKA (Bibb et al. 1999) and antagonizes the PKA/Thr34-DARPP-32/ protein phosphatase-1 cascade. Thus, DARPP-32 can act either as a phosphatase inhibitor or as a kinase inhibitor, depending on its relative state of phosphorylation.

Dopamine signaling is crucial in mediating the effects of psychostimulants such as cocaine (Nestler 2001), and DARPP-32 is critically involved in these effects. In striatal regions, acute cocaine administration induces an increase in
DARPP-32 phosphorylation at Thr34 associated with a relative decrease in Thr75 phosphorylation (Nishi et al. 2000; Rauggi et al. 2005), while repeated administration induces both behavioral sensitization and a tonic increase in phospho-Thr75 DARPP-32 levels, associated with a parallel decrease in phospho-Thr34 DARPP-32 levels (Bibb et al. 2001; Scheggi et al. 2004a,b). Repeated cocaine administration also induces the accumulation of $\Delta \mathrm{FosB}$ in the nucleus accumbens (NAc) that, in turn, increases the expression of cyclin-dependent kinase 5 (Cdk5) (Bibb et al. 2001). It has been reported that $\mathrm{Cdk} 5$ plays a counter-adaptive role in both the development and expression of cocaine-induced selfadministration and behavioral sensitization (Taylor et al.

Received March 31, 2007; revised manuscript received June 21, 2007; accepted June 25, 2007.

Address correspondence and reprint requests to Carla Gambarana, Department of Neuroscience, Pharmacology Unit, University of Siena, Via Moro, 4, 53100 Siena, Italy. E-mail: gambarana@unisi.it

Abbreviations used: Cdk5, cyclin-dependent kinase 5; DARPP-32, dopamine and cAMP-regulated phosphoprotein of $\mathrm{M}_{\mathrm{r}} 32$ 000; MPEP, 6-methyl-2-(phenylethynyl)-pyridine; mPFC, medial prefrontal cortex; NAc, nucleus accumbens; PBS, phosphate-buffered saline; PKA, cAMPdependent protein kinase; PP-1, protein phosphatase-1; Thr, threonine. 
2007). Mice carrying a point mutation derived by replacing the Thr residue with a non-phosphorylatable residue (alanine), at either the 34 or 75 position of DARPP-32, have been obtained (Svenningsson et al. 2003). An acute cocaine administration shows attenuated behavioral and neurochemical responses in Thr34 DARPP-32 mutant mice, that upon repeated cocaine administrations develop behavioral sensitization (Zachariou et al. 2006). On the contrary, Thr75 DARPP-32 mutant mice show a response to acute cocaine administration similar to that of wild-type mice, yet they do not develop behavioral sensitization (Zachariou et al. 2006). These data support the hypothesis that in cocaine-sensitized rats the tonic increase in phospho-Thr-75 DARPP-32 levels and decrease in phospho-Thr-34 DARPP-32 levels in the NAc is directly related to the condition of behavioral sensitization (Scheggi et al. 2004a,b).

Cocaine behavioral sensitization is induced and sustained by a multiplicity of neurochemical mechanisms, in which dopamine and glutamate play a central role. For example, NMDA receptor activity controls the development and the expression of cocaine sensitization (Karler et al. 1989; Wolf and Jeziorski 1993; Gambarana et al. 1998). Moreover, a precise interplay between glutamatergic transmission in the prefrontal cortex and the NAc decreases basal concentrations of extra-neuronal glutamate in the NAc, which results in a reduced inhibitory tonus of pre-synaptic mGluR $2 / 3$ and a consequent loss of regulatory feedback on synaptic glutamate release (Kalivas et al. 2003). Thus, lower basal levels of glutamate, combined with an increased release of synaptic glutamate in response to activation of prefrontal cortical afferents to the NAc, seem to underlie acute cocaine-induced behavioral changes in sensitized animals such as augmented motor activity (Vanderschuren and Kalivas 2000), enhanced place conditioning (Shippenberg et al. 1998), and self-administration relapse (Baker et al. 2003). Different metabotropic glutamate receptors appear to be involved in these mechanisms (Kalivas 2004; Kenny and Markou 2004).

Cocaine-induced behavioral sensitization is easily obtained by injecting animals with the drug, and it can be studied as a phenomenon, independent of the addictive properties of cocaine. The aim of the present study was to further characterize the modifications in DARPP-32/PKA pathway in cocaine-sensitized rats and to assess whether a temporal relationship exists between the observed pattern of neurochemical modifications and the condition of cocaine behavioral sensitization. Moreover, we were interested in the mechanisms that sustain the increased levels of phosphoThr75 DARPP-32 in the NAc of cocaine-sensitized rats. As an increase in phospho-Thr75 DARPP-32 levels has been associated with type I mGluRs activity in vitro (Liu et al. 2001) and it can be prevented by mGluR5 antagonism in vivo (Rauggi et al. 2005), we also investigated mGluR5 activity in cocaine-sensitized rats.

\section{Material and methods}

\section{Animals}

Experiments were carried out on male Sprague-Dawley rats (Charles River, Calco, Italy) weighing 125-150 g upon arrival in the vivarium. Animals were housed five per cage, kept in an environment maintained at a constant temperature and humidity with free access to food and water, and they were moved to a different cage or apparatus only for the time required for behavioral manipulation. A $12 \mathrm{~h}$ reverse light/dark cycle (7:00 AM lights off, 7 PM lights on) was used. Rats were allowed at least 1 week of habituation to the animal colony and when experimental procedures began they weighed 200-225 g. Experiments were carried out from 9:00 AM to 5:00 PM under a red light and controlled noise conditions in a testing room separated from and adjacent to the main animal room, under the same conditions of temperature and humidity. The procedures used in this study were in strict accordance with the European legislation on the use and care of laboratory animals (EEC Council Directive 86/609) and were approved by the University of Siena Ethics Committee.

\section{Induction of cocaine sensitization}

In previous studies we used repeated administrations of a high dose of cocaine to induce sensitization (cocaine $40 \mathrm{mg} / \mathrm{kg}$ i.p. on alternate days $\times 14$ days) (Scheggi et al. 2004a). However, as it was recently suggested that the modifications in DARPP-32 phosphorylation pattern observed in sensitized animals could be related to the high dose of cocaine to which rats were exposed rather than to the sensitization state (Hope et al. 2005), in the present study we utilized repeated administrations of a lower dose of cocaine: $10 \mathrm{mg} / \mathrm{kg}$ /day i.p. for eight consecutive days. Rats were individually placed in locomotor activity cages for $35 \mathrm{~min}$ after cocaine (or saline) administration to strengthen environmental contingencies and to favor the development of sensitization. At the end of the 8day protocol, animals were left undisturbed in their home cages for 10 days before the development of behavioral sensitization was assessed. Rats were then tested for their response to a cocaine challenge $(10 \mathrm{mg} / \mathrm{kg}$, i.p.) and their locomotor activity and stereotypies were recorded for $30 \mathrm{~min}$ (the first $5 \mathrm{~min}$ after injection were not taken into account).

The motor activity apparatus had eight compartments $(40 \times 45 \times 50 \mathrm{~cm})$ into which transparent Perspex cages (height $19 \mathrm{~cm}$, floor area $23 \times 33 \mathrm{~cm}$; Imetronic, Pessac, France) were placed. Motor activity was detected by a system of infrared photocell beams; the interruption of two photocell beams was recorded as an ambulatory activity count. A string of infrared photocell beams, placed at the height of $15 \mathrm{~cm}$, detected rearing activity. Stereotypies were scored by an experimenter blind to the experimental conditions, according to a published rating scale (Gambarana et al. 1998). A cumulative score reflecting the intensity of stereotypies during the 30 min observation period was assigned to each rat.

\section{Immunoblotting}

Rats were killed and brains were rapidly removed and cut into $1 \mathrm{~mm}$ slices using an ice-cold metal brain matrix (ASI Instruments, Inc., Warren, MI, USA). The medial prefrontal cortex (mPFC) and NAc were quickly dissected out from the slices that had been identified using the Atlas of Rat Brain as corresponding to plates 7-9 and 
(a)

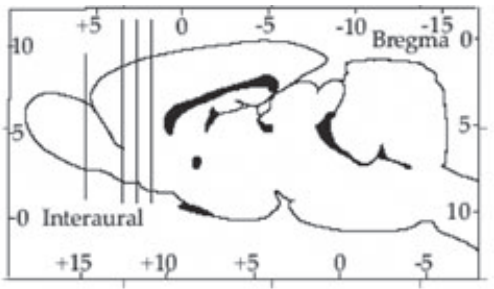

(b)
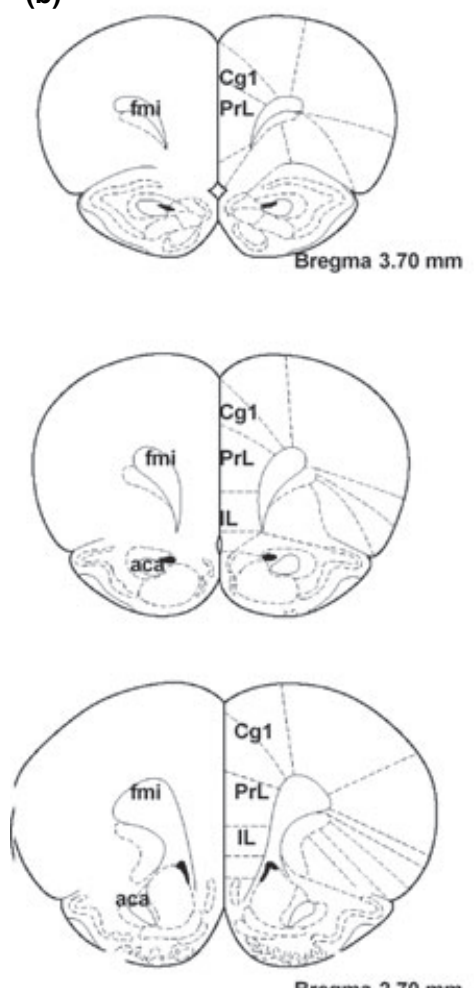

(c)
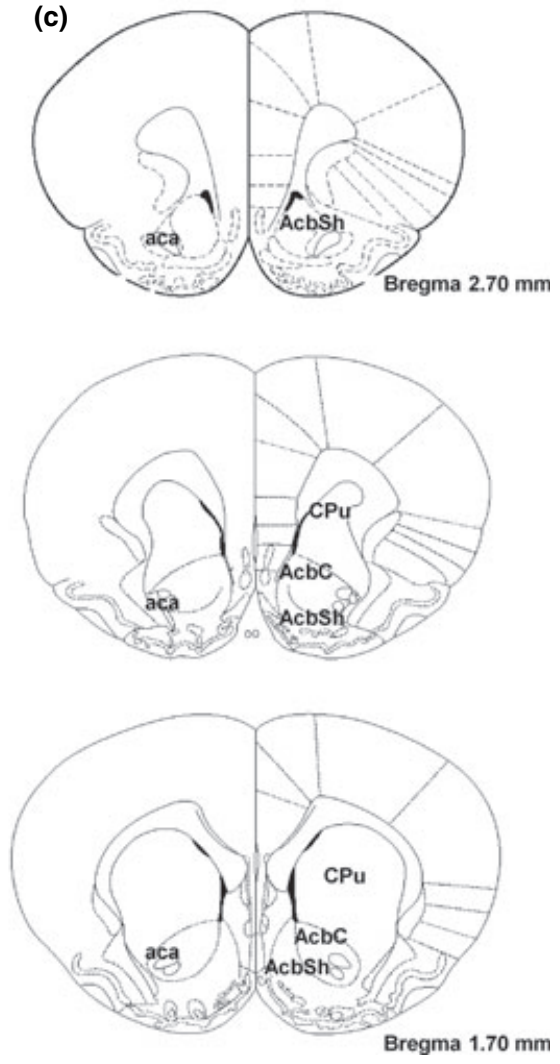

Fig. 1 Schematic representation, according to Paxinos and Watson (1998), of levels of brain slicing (a) for the MPFC (b) and NAc (c) regions as dissected for immunoblotting. $\mathrm{Cg}$, cingulate cortex, area 1; PrL, pre-limbic cortex; IL, infralimbic cortex; fmi, forceps minor corpus callosum; AcbC, accumbens nucleus, core; AcbSh, accumbens nucleus, shell; $\mathrm{CPu}$, caudate putamen; aca, anterior commissura, anterior part.
10-12, respectively (Paxinos and Watson 1998). The first slice was discarded and the mPFC (corresponding to the cingulate area, the pre-limbic cortex, and part of the infralimbic area) was dissected out from slice 2, approximately $3.7-2.7 \mathrm{~mm}$ from the bregma; the NAc (corresponding to the NAc shell and core) was dissected out from slice 3, approximately $2.7-1.7 \mathrm{~mm}$ from the bregma, and the anterior commissura was used as a point of reference (Fig. 1a, b, and c).

Tissues were then flash-frozen in liquid nitrogen and stored at $-80^{\circ} \mathrm{C}$ until assayed. Immunoblotting was performed as already described (Rauggi et al. 2005). Frozen tissue samples were prepared by solubilization in boiling $1 \%$ sodium dodecyl sulfate and $50 \mathrm{mmol} / \mathrm{L} \mathrm{NaFl}$. Aliquots containing $30 \mu \mathrm{g}$ of total protein were subjected to sodium dodecyl sulfate-polyacrylamide gel electrophoresis $(12 \%$ polyacrylamide gel for DARPP-32 and Cdk5; $7.5 \%$ polyacrylamide gel for $\alpha$-amino-3-hydroxy-5-methyl-4-isoxazolepropionic acid and NMDA receptor subunits) and transferred to nitrocellulose membranes. Small aliquots of the homogenate were retained for protein determination by a modified Lowry protein assay method (DC protein assay; BioRad Laboratories, Hercules, CA,
USA) (Lowry et al. 1951). Immunoblotting was carried out with phosphorylation-state-specific antibodies against phospho-Thr34 DARPP-32 and phospho-Thr75 DARPP-32 (Cell Signaling Technology, Beverly, MA, USA), phospho-Ser845 GluR 1 and phosphoSer897 NR 1 (Santa Cruz Biotechnology, Santa Cruz, CA, USA) or antibodies that are not phosphorylation-state-specific against total DARPP-32 (Cell Signaling Technology), total $\mathrm{GluR}_{1}$ and $\mathrm{NR}_{1}$ (Santa Cruz Biotechnology), and Cdk5 (Santa Cruz Biotechnology). Antibody binding was detected using a chemiluminescence detection system (Pierce Biotechnology Inc., Rockford, IL, USA) and quantified with the Versa Doc 1000 Imaging System (BioRad Laboratories). Samples containing the same amount of total proteins from control and treated rats were run on the same immunoblots and then analyzed together. For each experiment, values obtained from experimental groups were calculated as the percentage of their respective control values.

The total amount of DARPP-32 was unmodified, compared to the control group, in the mPFC and NAc in all experiments (data not shown). 


\section{Immunohistochemistry}

Immunohistochemistry was performed as previously described (Scheggi et al. 2004b). Briefly, coronal sections (35 $\mu \mathrm{m})$, taken from brain levels corresponding to plates 10-13 of the Atlas of Rat Brain (Paxinos and Watson 1998), were cut on a cryostat and collected in cold phosphate-buffered saline (PBS). Free-floating sections were pre-treated for 10 min with $1 \%$ hydrogen peroxide in PBS and for $1 \mathrm{~h}$ with blocking solution containing $0.2 \%$ Triton $\mathrm{X}-100$ and $2 \%$ normal goat serum in PBS. Sections were incubated at $4^{\circ} \mathrm{C}$ for $48 \mathrm{~h}$ with anti-FosB antiserum $(1: 250)$ (Santa Cruz Biotechnology) in PBS containing $0.2 \%$ Triton X-100 and $2 \%$ normal goat serum. This antibody was raised against an $\mathrm{N}$-terminal peptide that is common to full-length FosB and $\Delta \mathrm{FosB}$ and, in preliminary immunoblotting experiments, we detected two distinct immunoreactive bands with an apparent molecular weight of 46 and 32-37 kDA (data not shown), corresponding to full length Fos and $\Delta$ FosB, respectively (Nakabeppu and Nathans 1991; McClung et al. 2004). After several PBS washes, sections were incubated for $1 \mathrm{~h}$ with biotinylated secondary antibody at $37^{\circ} \mathrm{C}$ (Vectastain EliteABCKit; Vector Laboratories Inc., Burlinghame, CA, USA). After further PBS washes, sections were incubated with biotin-streptavidin-peroxidase complex and the peroxidase reaction product was visualized by 3,3'-diaminebenzidine (Vectastain EliteABCKit; Vector Laboratories Inc.). Sections were mounted onto electrostatic superfrost glass slides, dehydrated, and coverslipped. FosB/ $\Delta$ FosB positive cells were counted with a light microscope (Eclipse 50i; Nikon Instruments S.P.A., Sesto Fiorentino, Italy) equipped with a digital camera (DS-5M; Nikon); using NIH IMAGE software (Bethesda, MD, USA), a defined square was placed over the NAc and immunoreactive positive cells were counted by an experimenter blind to the experimental conditions. To verify the accuracy of automatic counts, intermittently grains were counted manually. Bilateral grain counts from three consecutive sections were taken to obtain the average value from each rat, and sections taken from five rats in each experimental group were used.

\section{Experimental protocols}

In order to assess whether behavioral expression of sensitization was a relatively long-lasting phenomenon, and whether defined neuro- chemical modifications associated with repeated cocaine exposure were temporally related to the expression of sensitization, groups of rats were exposed to the sensitization protocol, and then tested and killed at different times after the last cocaine exposure. Two different intervals (washout periods) between assessment of the development of sensitization and behavioral testing and neurochemical determinations were selected (10 days and 3 months). In order to group behavioral tests and animal sacrifice in a few week interval, experiment $\mathrm{B}$ began about 2 months before experiment $\mathrm{A}$.

Experiment A-1 - Characterization of neurochemical modifications accompanying the condition of cocaine behavioral sensitization after a short-term washout

Rats underwent the sensitization protocol (repeated cocaine, $n=15)$, or received saline $(1 \mathrm{~mL} / \mathrm{kg} /$ day, i.p., for 8 days, repeated saline, $n=25$ ). After a 10-day washout, the rats exposed to the sensitization protocol and 10 rats from the control group were challenged with cocaine (10 mg/kg, i.p.) to assess the development of behavioral sensitization. The behavioral response to the challenge was recorded for $30 \mathrm{~min}$ (Table 1). Ten days later, five rats in the repeated cocaine group and five of the repeated saline rats not yet exposed to cocaine were administered cocaine, five more rats from each of these groups were administered saline. Thirty-five minutes after cocaine or saline administration, all 20 rats were killed.

In the $\mathrm{mPFC}$ and NAc dissected out from brains of repeated saline and repeated cocaine rats, exposed to saline or cocaine administration, the phosphorylation pattern of DARPP-32 and the levels of Cdk5, GluR ${ }_{1}$ and phospho-GluR ${ }_{1}, \mathrm{NR}_{1}$, and phospho-NR were determined. In the remaining repeated saline and repeated cocaine rats ( $n=5$ for each group), the expression of $\Delta \mathrm{FosB}$ in the NAc was examined.

Experiment A-2 - Challenge with different doses of cocaine in sensitized rats: behavioral response and modifications in the levels of phosphorylation of DARPP-32, and GluR 1 and $\mathrm{NR}_{1}$ subunits

Rats were exposed to the cocaine sensitization protocol (repeated cocaine, $n=15)$ or to saline treatment $(1 \mathrm{~mL} / \mathrm{kg} /$ day, i.p. for 8 days, repeated saline, $n=21)$. After a 10 -day washout, the development
Table 1 Behavioral responses to cocaine challenge

\begin{tabular}{|c|c|c|c|c|c|}
\hline & Group & $n$ & $\begin{array}{l}\text { Stereotypy } \\
\text { score }\end{array}$ & Rearing number & $\begin{array}{l}\text { Horizontal } \\
\text { locomotor } \\
\text { activity }\end{array}$ \\
\hline \multicolumn{6}{|c|}{ Experiment $\mathrm{A}$} \\
\hline \multirow[t]{2}{*}{1} & Repeated saline & 10 & $2.2 \pm 0.3$ & $89.5 \pm 23.8$ & $117.2 \pm 19.0$ \\
\hline & Repeated cocaine & 15 & $4.4 \pm 0.1^{\star * *}$ & $197.9 \pm 9.0^{\star \star \star}$ & $543.6 \pm 49.3^{\star \star \star}$ \\
\hline \multirow[t]{2}{*}{2} & Repeated saline & 6 & $1.7 \pm 0.2$ & $97.0 \pm 32.3$ & $167.2 \pm 56.6$ \\
\hline & Repeated cocaine & 15 & $3.9 \pm 0.1^{\star \star \star}$ & $178.9 \pm 24.5$ & $424.7 \pm 81.9$ \\
\hline \multirow[t]{2}{*}{3} & Repeated saline & 5 & $1.9 \pm 0.4$ & $88.4 \pm 43.0$ & $105.4 \pm 30.4$ \\
\hline & Repeated cocaine & 32 & $4.4 \pm 0.2$ & $149.1 \pm 18.8$ & $285.5 \pm 50.0$ \\
\hline \multicolumn{6}{|c|}{ Experiment B } \\
\hline & Repeated saline & 5 & $1.9 \pm 0.6$ & $77.8 \pm 32.2$ & $175.6 \pm 31.2$ \\
\hline & Repeated cocaine & 20 & $4.6 \pm 0.1^{\star \star \star}$ & $216.5 \pm 10.9^{\star \star \star}$ & $538.9 \pm 53.3^{\star \star}$ \\
\hline & Repeated saline $3 m$ & 5 & $1.5 \pm 0.4$ & $153.6 \pm 28.1$ & $172.4 \pm 39.8$ \\
\hline & Repeated cocaine $_{3 m}$ & 8 & $2.1 \pm 0.2^{\S \S \S}$ & $190.8 \pm 28.4$ & $531.3 \pm 87.6^{*}$ \\
\hline
\end{tabular}

${ }^{*} p<0.05,{ }^{* *} p<0.01,{ }^{* *} p<0.001$ compared to the respective repeated saline groups (unpaired $t$-test); ${ }^{\S \S} p<0.001$ compared to the repeated cocaine group. 
of sensitization was ascertained in all rats exposed to the sensitization protocol and in six repeated saline rats (Table 1). Ten days later, rats in the repeated cocaine and repeated saline groups received an acute injection of saline $(1 \mathrm{~mL} / \mathrm{kg})$ or cocaine $(10$ or $20 \mathrm{mg} / \mathrm{kg}$ ), ( $n=5$ for each subgroup). The behavioral response to the challenge was recorded and rats were then killed $35 \mathrm{~min}$ after the injection. The DARPP-32 phosphorylation pattern and phosphoGluR $_{1}$ and phospho-NR $\mathrm{NR}_{1}$ levels were determined in the NAc.

Experiment A-3 - Acute mGluR5 inhibition in cocainesensitized rats: behavioral effects and modifications in levels of phosphorylation of DARPP-32, and GluR $\mathbf{1}_{1}$ and $\mathrm{NR}_{\mathbf{1}}$ subunits

Rats were exposed to the cocaine sensitization protocol (repeated cocaine, $n=32)$ or to saline treatment $(1 \mathrm{~mL} / \mathrm{kg} /$ day, i.p. for 8 days, repeated saline, $n=28$ ). After a 10 -day washout, the development of sensitization was assessed in all rats exposed to the sensitization protocol and in a group of five repeated saline rats (Table 1). Ten days later, rats in each group were divided into two subgroups, one receiving a saline injection $(1 \mathrm{~mL} / \mathrm{kg}$, i.p.) and the other receiving a selective antagonist of mGluR5 receptors, 6-methyl-2-(phenylethynyl)-pyridine, MPEP ( $5 \mathrm{mg} / \mathrm{kg}$, i.p.) $1 \mathrm{~h}$ before sacrifice. Twentyfive minutes after saline or MPEP administration, rats in each subgroup received a saline $(1 \mathrm{~mL} / \mathrm{kg}$, i.p. $)$ or cocaine injection $(10 \mathrm{mg} / \mathrm{kg}$, i.p. $),(n=4-8$ rats in each subgroup of the repeated saline and repeated cocaine groups) and were placed in the motor activity apparatus. Locomotor activity and stereotypy scores were recorded for $30 \mathrm{~min}$, then rats were killed and the pattern of DARPP-32 phosphorylation and levels of phospho-GluR ${ }_{1}$ and phospho- $\mathrm{NR}_{1}$ in the mPFC and NAc were determined. A group of five repeated saline rats never exposed to behavioral test were used as the reference control group for immunoblotting experiments.

The dose of MPEP was selected on the basis of previous studies (Paterson et al. 2003) and our own study showing that $5 \mathrm{mg} / \mathrm{kg}$ of MPEP did not induce overt modifications in the spontaneous behavior of rats (Rauggi et al. 2005).

\section{Experiment B - Assessment of behavioral sensitization to} cocaine and of the neurochemical modifications associated with repeated cocaine exposure after a long-term washout

Rats underwent either the cocaine sensitization protocol $(10 \mathrm{mg} / \mathrm{kg} /$ day, i.p., $n=20)$ or were treated with saline $(1 \mathrm{~mL} / \mathrm{kg} /$ day, i.p., $n=20$ ) for 8 days. After a 10-day washout all cocaine-treated rats and five saline-treated rats were challenged with cocaine $(10 \mathrm{mg} / \mathrm{kg}$, i.p.) and motor activity and stereotypy scores were determined (Table 1). Then, rats that had developed sensitization (repeated cocaine $_{3 m}, n=20$ ) and the saline-treated control rats never exposed to cocaine (repeated saline $e_{3 m}, n=15$ ) were kept in the vivarium for 3 months, where they underwent frequent handling. At the end of this period, to verify whether behavioral sensitization was still fully expressed in previously sensitized rats, eight rats in the repeated cocaine $_{3 m}$ group, and five rats in the repeated saline ${ }_{3 m}$ group underwent a cocaine challenge $(10 \mathrm{mg} / \mathrm{kg}$, i.p.). Thirty-five minutes after cocaine administration rats in the two groups were killed, along with five repeated saline $_{3 m}$ and five repeated cocaine $3 m$ rats not exposed to the final cocaine challenge. The mPFC and NAc were dissected out to determine the phosphorylation pattern of DARPP32 and the levels of Cdk5, GluR 1 and phospho-GluR $1, \mathrm{NR}_{1}$, and phospho- $\mathrm{NR}_{1}$. The remaining repeated saline $_{3 m}$ and repeated cocaine $_{3 m}$ rats not exposed to the cocaine challenge ( $n=5$ for each group) were killed at the same time as five rats in the repeated saline and five rats in the repeated cocaine groups from the shortterm washout experiment (experiment $\mathrm{A}$ ) and the expression of $\Delta \mathrm{FosB}$ in the NAc was examined in the four groups.

\section{Drugs}

Cocaine and MPEP were dissolved in deionized/distilled water and injected in a volume of $1 \mathrm{~mL} / \mathrm{kg}$ rat body weight. Cocaine was purchased from Macfarlan Smith Limited (Edinburgh, UK) and MPEP from Tocris Cookson Ltd (Avonmouth, UK). All chemicals were purchased from commercial sources.

\section{Statistical analysis}

Statistical analyses were performed using commercially available software (Prism 4.0a, GraphPad Software Inc., San Diego, CA, USA). All data are expressed as mean \pm SEM. Statistical comparisons of behavioral measures were made by unpaired $t$-test or, when more than two groups were analyzed, by ANOva followed by Dunnett's test when $p<0.05$. Western blot and immunohistochemical data were analyzed using ANOvA and post hoc analysis was performed by Bonferroni's test when $p<0.05$, unless otherwise specified.

\section{Results}

\section{Experiment A-1 - Characterization of neurochemical modifications accompanying the condition of cocaine behavioral sensitization}

Rats exposed to the protocol of cocaine sensitization and rats in the control group received a cocaine challenge after a 10-day washout. The challenge elicited an intense motor response in rats exposed to the sensitization protocol: stereotypy scores and locomotor activity were significantly higher in this group (repeated cocaine) than in the repeated saline group $(p<0.001$ for stereotypy scores, rearing number, and horizontal locomotion, unpaired $t$-test; Table 1).

This experiment was aimed at examining a pattern of neurochemical modifications that accompany the behavioral expression of cocaine sensitization after a short-term washout. Moreover, to examine whether some of the neurochemical variables studied (phosphorylation pattern of DARPP-32, phosphorylation levels of $\mathrm{GluR}_{1}$, and $\mathrm{NR}_{1}$ subunits) would change in response to acute cocaine administration in sensitized rats as they do in control rats, five animals in the repeated saline and repeated cocaine groups were exposed to a cocaine challenge and then killed 35 min later.

\section{Western blot analysis in the mPFC: DARPP-32 phosphorylation pattern}

In the mPFC, phospho-Thr75 DARPP-32 levels were significantly different between rats in the repeated saline and repeated cocaine groups $\left(F_{3,19}=19.22, p<0.001\right)$. Post hoc analysis demonstrated that in repeated saline and 


\begin{abstract}
Fig. 2 Levels of phospho-Thr75 and phospho-Thr34 DARPP-32, phosphoGluR $_{1}$, and phospho-NR 1 in the mPFC of control (repeated saline) and cocaine-sensitized (repeated cocaine) rats, after a saline $(1 \mathrm{~mL} / \mathrm{kg})$ or cocaine $(10 \mathrm{mg} / \mathrm{kg}$, i.p.) challenge, administered after a 10-day washout. Levels of phospho-Thr75 DARPP32 (a), phospho-Thr34 DARPP-32 (b), phospho-GluR $\mathrm{R}_{1}$ (c), and phospho-NR $\mathrm{N}_{1}$ (d), shown in graphical format in the upper panels, are expressed as mean \pm SEM percentage of values in the saline-treated control group. Representative immunoblots are presented in the lower panels. ${ }^{* \star *} p<0.001,{ }^{* *} p<0.01,{ }^{*} p<0.05$ versus the saline-treated repeated saline group; ${ }^{\S} p<0.05, \quad{ }^{\S} p<0.01$ versus the salinetreated repeated cocaine group (Bonferroni's test).
\end{abstract}

(a)
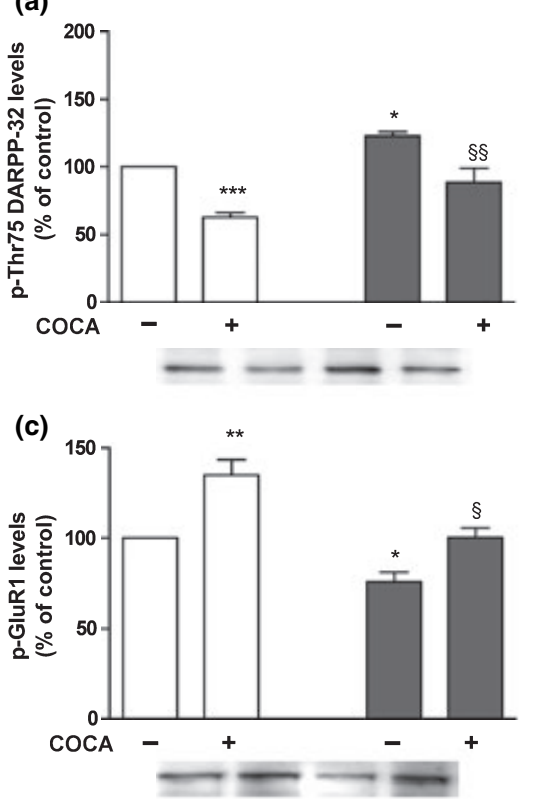

MPFC

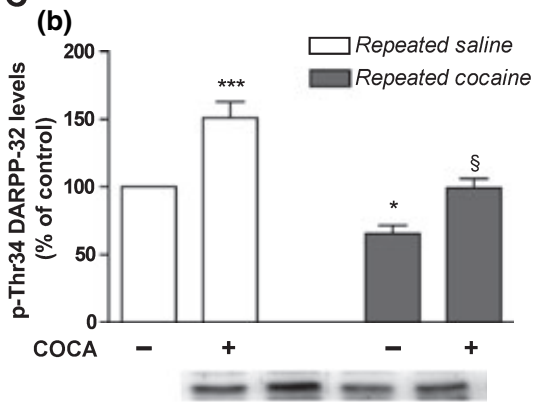

(d)

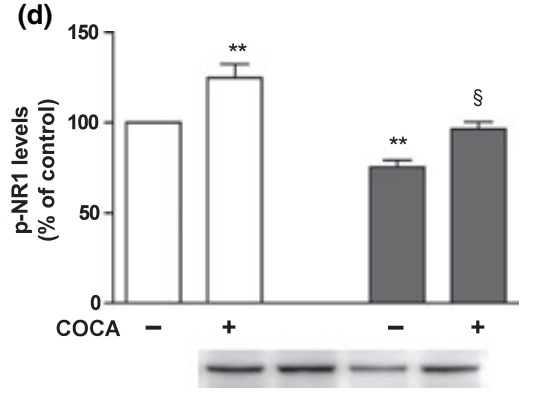

repeated cocaine rats not exposed to the cocaine challenge, phospho-Thr75 DARPP-32 levels were higher in the repeated cocaine group $(p<0.05)$. After the challenge, phospho-Thr75 DARPP-32 levels decreased in the repeated saline group and in the repeated cocaine group, compared to their respective saline-treated groups $(p<0.001$ and $p<0.01$, respectively; Fig. 2a). Phospho-Thr34 DARPP-32 levels were significantly different between groups $\left(F_{3,19}=22.76 ; p<0.001\right)$, and post hoc analysis demonstrated that in rats not exposed to the challenge phosphoThr34 DARPP-32 levels were lower in the repeated cocaine group compared to the repeated saline group $(p<0.05)$. After cocaine challenge, phospho-Thr34 DARPP-32 levels increased in the repeated saline and in the repeated cocaine group, compared to their respective saline-treated groups $(p<0.001$ and $p<0.05$, respectively; Fig. 2b). The phosphorylation levels of two other PKA substrates, $\mathrm{GluR}_{1}$ and $\mathrm{NR}_{1}$ subunits, were then measured.

\section{Levels of GluR 1 and $\mathrm{NR}_{1}$ subunits and of their phosphorylated forms}

The levels of GluR $R_{1}$ and $\mathrm{NR}_{1}$ subunits were similar in rats in the repeated cocaine and repeated saline groups $\left(\mathrm{GluR}_{1}\right.$, repeated cocaine $=102.3 \pm 7.4 \%$ of repeated saline group; $\mathrm{NR}_{1}$, repeated cocaine $=111.8 \pm 10.6 \%$ of repeated saline group, unpaired $t$-test). GluR $\mathrm{G}_{1}$ and $\mathrm{NR}_{1}$ subunit phosphorylation levels in rats of the repeated saline and repeated cocaine groups, administered saline or cocaine, were different (phospho-GluR $1, \quad F_{3}, \quad 19=17.58, \quad p<0.001$; phospho- $\left.\mathrm{NR}_{1}, \mathrm{~F}_{3,19}=18.21, p<0.001\right)$. In particular, in saline-treated rats, phospho-GluR $R_{1}$ and phospho-NR $\mathrm{N}_{1}$ levels were lower in the repeated cocaine group than in the repeated saline group (phospho-GluR $\mathrm{G}_{1}, p<0.05$; phospho$\mathrm{NR}_{1}, p<0.01$; Fig. $2 \mathrm{c}$ and d). After cocaine challenge, the levels of phosphorylation of the GluR $\mathrm{R}_{1}$ and $\mathrm{NR}_{1}$ subunits increased in both repeated saline and repeated cocaine groups, compared to their respective saline-treated groups (phospho-GluR,$p<0.01$ for the repeated saline group, $p<0.05$ for the repeated cocaine group; phospho-NR $\mathrm{N}_{1}$, $p<0.01$ for the repeated saline group, $p<0.05$ for the repeated cocaine group; Fig. $2 \mathrm{c}$ and d).

\section{Cdk5 levels}

As repeated cocaine administration increases Cdk5 expression in striatal areas, we investigated the existence of a possible link between this increase and the condition of cocaine sensitization. After a 10-day washout, the levels of expression of Cdk5 were higher in rats in the repeated cocaine group than in rats in the repeated saline group (repeated cocaine $=138+4.8 \%$ of control value, $p<0.001$, unpaired $t$-test).

\section{Western blot analysis in the NAc: DARPP-32 phosphorylation pattern}

In the NAc, levels of phospho-Thr75 DARPP-32 showed a significant difference between groups $\left(F_{3,19}=56.45\right.$, $p<0.001$ ). In saline-treated rats, phospho-Thr75 DARPP32 levels were higher in the repeated cocaine group than in the repeated saline group $(p<0.001$; Fig. $3 a)$. In rats exposed to the challenge, levels of phospho-Thr75 DARPP-32 decreased in the repeated saline group $(p<0.001)$, but were unmodified in the repeated cocaine group, 


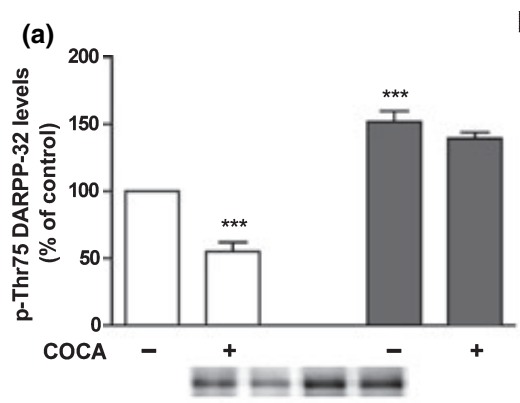

NAc (b)
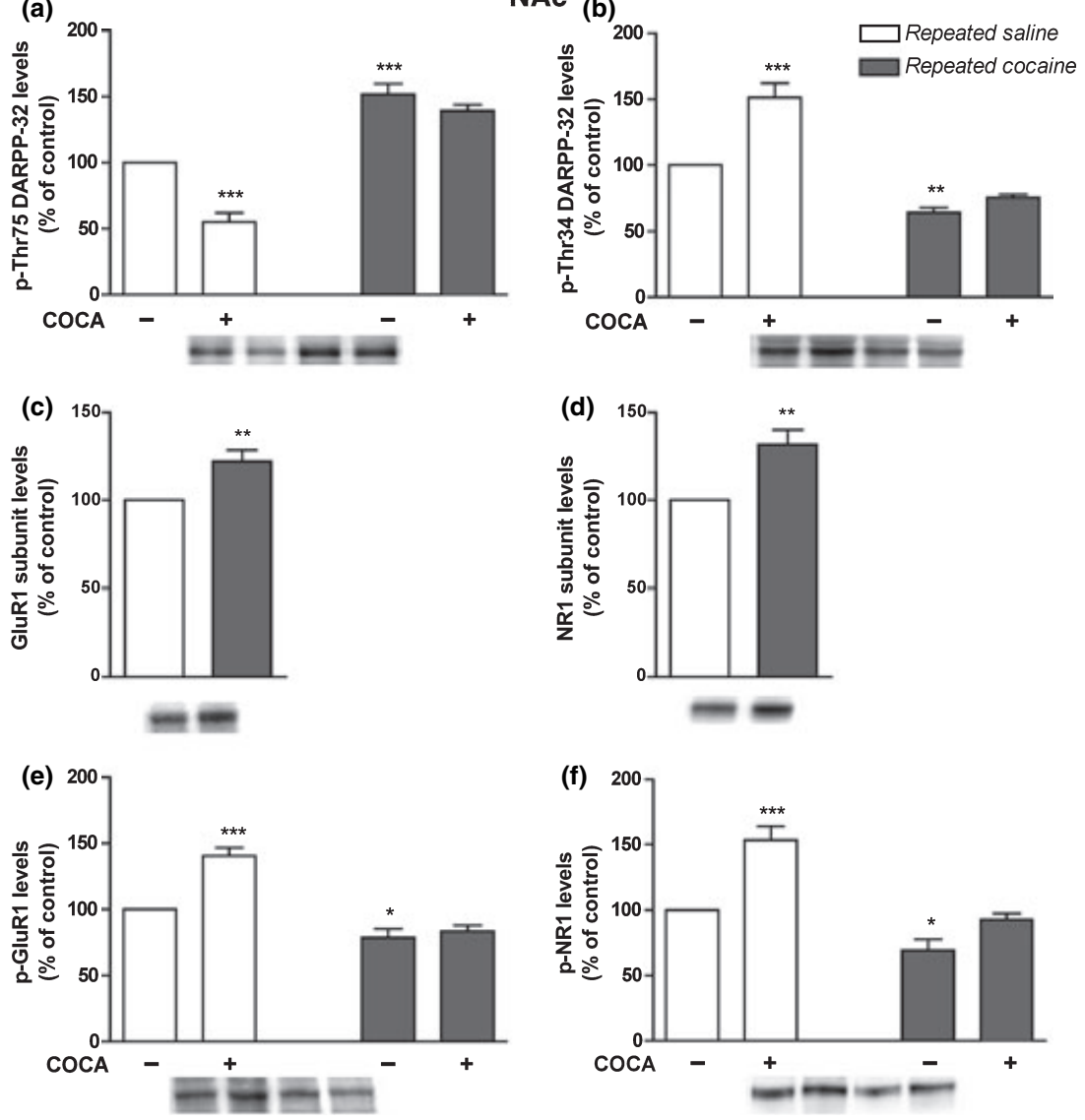
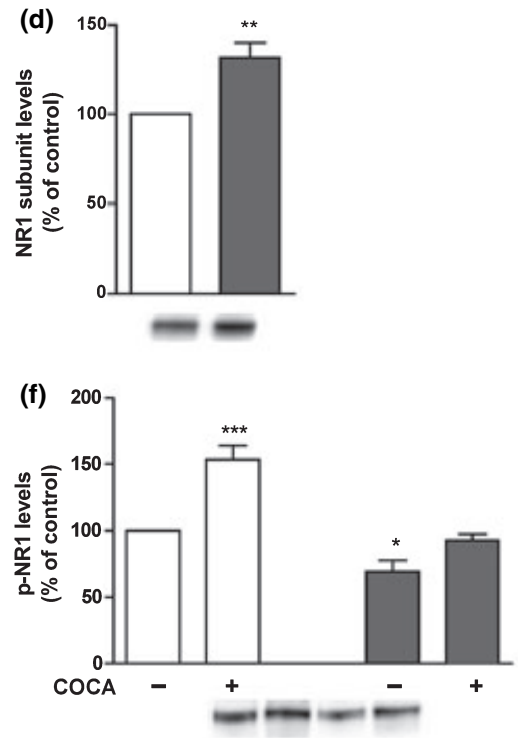

Fig. 3 Levels of phospho-Thr75 and phospho-Thr34 DARPP-32, GluR, $\mathrm{NR}_{1}$, phospho-GluR 1 , and phospho-NR $\mathrm{N}_{1}$ in the NAc of control (repeated saline) and cocaine-sensitized (repeated cocaine) rats, after a saline $(1 \mathrm{~mL} / \mathrm{kg})$ or cocaine $(10 \mathrm{mg} /$ $\mathrm{kg}$, i.p.) challenge, administered after a 10day washout. Levels of phospho-Thr75 DARPP-32 (a), phospho-Thr34 DARPP-32 (b), GluR 1 (c), NR 1 (d), phospho-GluR 1 (e), and phospho-NR $\mathrm{N}_{1}$ (f) are expressed as mean \pm SEM percentage of values in the saline-treated control group. Representative immunoblots are presented in the lower panels. ${ }^{\star \star *} p<0.001,{ }^{* \star} p<0.01,{ }^{\star} p<0.05$ versus the saline-treated repeated saline group (Bonferroni's test). compared to their respective saline-treated groups (Fig. 3a). Levels of phospho-Thr34 DARPP-32 were also significantly different between groups $\left(F_{3,19}=44.77, p<0.001\right)$. In saline-treated rats, phospho-Thr34 DARPP-32 levels were lower in the repeated cocaine group than in the repeated saline group ( $p<0.01$; Fig. $3 \mathrm{~b})$. Moreover, in rats exposed to cocaine challenge, levels of phospho-Thr34 DARPP-32 increased in the repeated saline group $(p<0.001)$, but not in the repeated cocaine group, compared to their respective saline-treated groups (Fig. 3b).

\section{Levels of GluR 1 and $\mathrm{NR}_{1}$ subunits and of their phosphorylated forms}

The expression of both $\mathrm{GluR}_{1}$ and $\mathrm{NR}_{1}$ subunits was higher in rats in the repeated cocaine group than in rats in the repeated saline group $(p<0.01$ and $p<0.01$, respectively, unpaired $t$-test; Fig. $3 \mathrm{c}$ and d). One-way ANOvA analysis of the phosphorylation levels of GluR 1 and $\mathrm{NR}_{1}$ in rats of the repeated saline and repeated cocaine groups indicated a significant difference between groups for both phosphorylated subunits (phospho-GluR,$F_{3,19}=30.53$, $p<0.001 ;$ phospho-NR $\left., \quad F_{3,19}=25.68, p<0.001\right)$. In particular, in saline-treated rats, phospho-GluR ${ }_{1}$, and
phospho-NR $\mathrm{NR}_{1}$ levels were lower in the repeated cocaine group than in the repeated saline group (phospho-GluR 1 , $p<0.05$; phospho-NR $1, p<0.05$; Fig. $3 \mathrm{e}$ and f). After cocaine challenge, the levels of the phosphorylated GluR $_{1}$ and $\mathrm{NR}_{1}$ subunits increased in rats of the repeated saline group, compared to their saline-treated group $(p<0.001$ for both phospho-GluR $\mathrm{R}_{1}$ and phospho-NR $\mathrm{N}_{1}$ ), but they were unchanged in rats of the repeated cocaine group (Fig. $3 \mathrm{e}$ and $\mathrm{f}$ ).

\section{Cdk5 levels}

Levels of expression of Cdk5 in the NAc were higher in rats in the repeated cocaine group than rats in the repeated saline group (repeated cocaine $=163+13.3 \%$ of control value, $p<0.01$, unpaired $t$-test).

The results of experiment A-1 showed consistent modifications in the phosphorylation pattern of DARPP-32, GluR 1 and $\mathrm{NR}_{1}$ subunits, and in Cdk5 levels in the mPFC and NAc of sensitized rats after a 10-day washout. Moreover, another hallmark of the condition of cocaine sensitization emerged, i.e. the lack of effect of an acute cocaine administration on the phosphorylation levels of DARPP-32, GluR ${ }_{1}$, and $\mathrm{NR}_{1}$ in the NAc. 
Experiment A-2 - Challenge with different doses of cocaine in sensitized rats: behavioral response and modifications in the levels of phosphorylation of DARPP-32, and GluR ${ }_{1}$ and $\mathrm{NR}_{1}$ subunits

In order to examine whether the lack of modifications in DARPP-32 phosphorylation pattern after an acute cocaine challenge was the expression of reduced responsiveness of the dopamine $\mathrm{D}_{1}$ receptor/cAMP/PKA pathway to increased dopamine levels, 10 days after the assessment of sensitization (Table 1), sensitized and control rats were challenged with saline $(1 \mathrm{~mL} / \mathrm{kg})$ or cocaine $(10$ or $20 \mathrm{mg} / \mathrm{kg}$ i.p.) and then killed $35 \mathrm{~min}$ after injection. At the dose of $10 \mathrm{mg} / \mathrm{kg}$, cocaine induced higher stereotyped and locomotor activity in sensitized rats than in control rats, as expected. At the dose of $20 \mathrm{mg} / \mathrm{kg}$, cocaine elicited similar stereotyped and locomotor activity in rats in the repeated saline and repeated cocaine groups (data not shown).

Levels of expression and/or phosphorylation of DARPP32, $\mathrm{GluR}_{1}$, and $\mathrm{NR}_{1}$ were determined only in the NAc. In order to confirm that these variables were modified in cocaine-sensitized rats, as observed in experiment A-1, western blot data of all the six groups were analyzed by ANOVA and when $p<0.05$ Bonferroni's test was used to
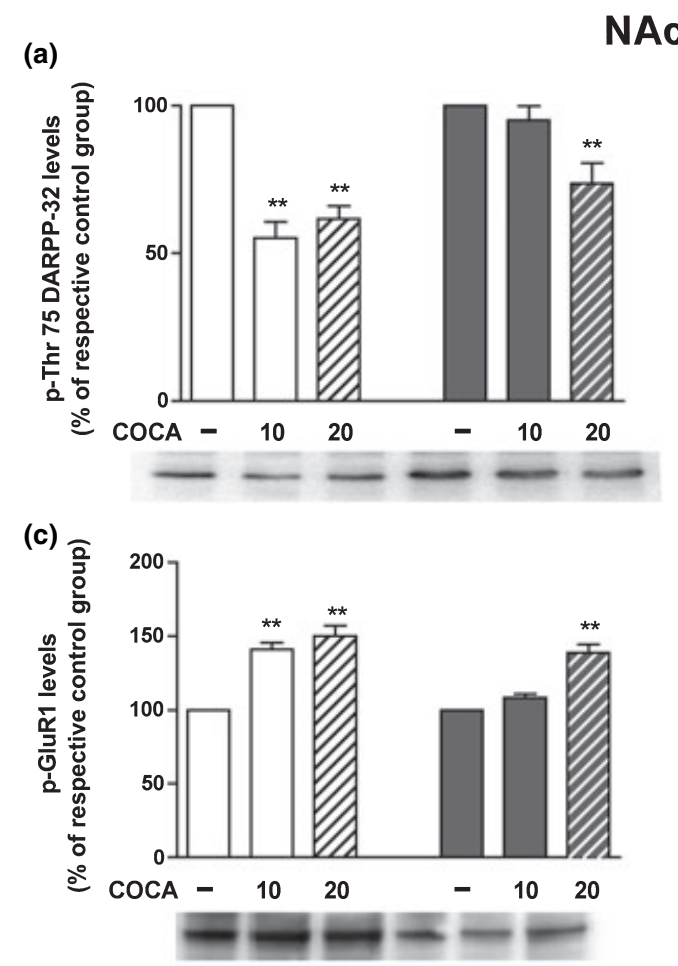

Fig. 4 Levels of phospho-Thr75 and phospho-Thr34 DARPP-32, phospho-GluR $\mathrm{R}_{1}$, and phospho-NR $\mathrm{NR}_{1}$ in the NAc of control (repeated saline) and cocaine-sensitized (repeated cocaine) rats, after a saline $(1 \mathrm{~mL} / \mathrm{kg}$ ) or cocaine (10 or $20 \mathrm{mg} / \mathrm{kg}$, i.p.) challenge, administered after a 10-day washout. Levels of phospho-Thr75 DARPP-32 (a), assess whether the levels of the variables studied were different between the repeated saline and repeated cocaine saline-treated groups. Data were then recalculated in order to express levels in the repeated saline and repeated cocaine challenged groups as $100 \%$ of their respective controls (the saline-treated group), and then analyzed by ANOvA and post hoc Dunnett's test. Analysis of phospho-Thr75 DARPP-32 levels in all six subgroups of rats showed a significant difference $\left(F_{5,29}=36.48, p<0.001\right)$; in particular, post hoc analysis confirmed that in saline-treated rats levels of phospho-Thr75 DARPP-32 were higher in the repeated cocaine than in the repeated saline group $(p<0.001)$. When data were recalculated in order to express phospho-Thr75 DARPP-32 levels in the repeated saline and repeated cocaine challenged groups as the percentage of their respective controls (the saline-treated groups), statistical analysis indicated a significant difference between phosphoThr75 DARPP-32 levels in the three subgroups of repeated saline rats $\left(F_{2,14}=34.53, p<0.001 ;\right.$ Fig. $\left.4 \mathrm{a}\right)$. In fact, acute cocaine administration induced a decrease in phospho-Thr75 DARPP-32 levels compared to levels in saline-treated rats, and this effect was already maximal at the $10 \mathrm{mg} / \mathrm{kg}$ dose $(p<0.01$, for both the 10 and $20 \mathrm{mg} / \mathrm{kg}$ cocaine doses;
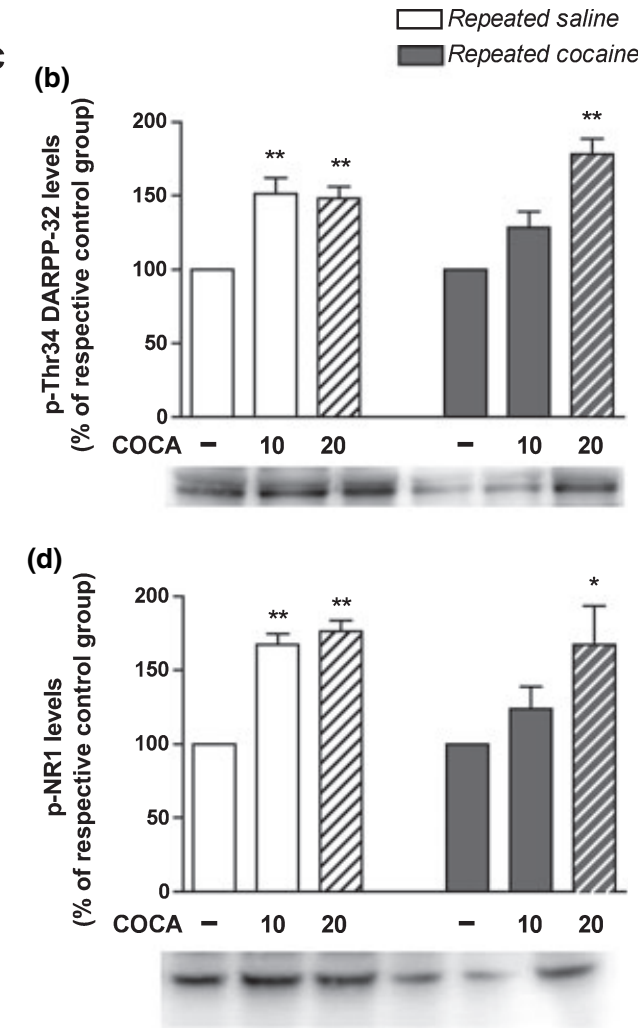

phospho-Thr34 DARPP-32 (b), phospho-GluR 1 (c), and phospho-NR (d) are expressed as mean \pm SEM percentage of values in the salinetreated respective control groups. Representative immunoblots are presented in the lower panels. ${ }^{* *} p<0.01,{ }^{*} p<0.05$ versus the salinetreated respective control groups (Bonferroni's test). 
Fig. 4a). In rats in the repeated cocaine group, a significant difference in the levels of phospho-Thr75 DARPP-32 was demonstrated between subgroups $\left(F_{2,14}=8.25, p<0.01\right.$; Fig. 4a). As observed in the previous experiment, the $10 \mathrm{mg} /$ $\mathrm{kg}$ cocaine challenge did not modify the pattern of DARPP32 phosphorylation in sensitized rats. However, after the $20 \mathrm{mg} / \mathrm{kg}$ cocaine challenge, phospho-Thr75 DARPP-32 levels decreased compared to levels in the saline-treated subgroup ( $p<0.01$; Fig. $4 \mathrm{a}$ ). Phospho-Thr34 DARPP-32 levels in the six subgroups of rats were significantly different $\left(F_{5,29}=31.14, p<0.001\right)$; in particular, post hoc analysis confirmed that levels of phospho-Thr34 DARPP-32 were lower in the repeated cocaine than in the repeated saline group $(p<0.001)$. When data were recalculated in order to express phospho-Thr34 DARPP-32 levels in the repeated saline and repeated cocaine challenged groups as the percentage of their respective saline-treated groups, statistical analysis indicated a significant difference in phospho-Thr34 DARPP-32 levels in the three subgroups of repeated saline rats $\left(F_{2,14}=14.42, p<0.001\right)$. Acute cocaine administration induced an increase in phospho-Thr34 DARPP-32 levels compared to levels in the repeated saline saline-treated rats, and this effect was already maximal at the $10 \mathrm{mg} / \mathrm{kg}$ dose ( $p<0.01$ for both doses; Fig. 4b). In rats in the repeated cocaine group, a significant difference in the levels of phospho-Thr34 DARPP-32 was also demonstrated between subgroups $\left(F_{2,14}=20.51, p<0.001\right)$. As observed in the previous experiment, the $10 \mathrm{mg} / \mathrm{kg}$ cocaine challenge did not modify phospho-Thr34 DARPP-32 levels in cocaine-sensitized rats. However, after the $20 \mathrm{mg} / \mathrm{kg}$ cocaine challenge, levels of phospho-Thr34 DARPP-32 increased compared to levels in the repeated cocaine saline-treated subgroup ( $p<0.01$; Fig. 4b).

The levels of phospho-GluR $R_{1}$ and phospho-NR $\mathrm{N}_{1}$ subunits in the repeated saline group were significantly different between subgroups (phospho-GluR,$\quad F_{5,29}=38.85$, $p<0.001$; phospho-NR $\left.1, F_{5,27}=73.29, p<0.001\right)$; in particular, post hoc analysis confirmed that levels of both phosphorylated subunits were higher in the repeated saline than in the repeated cocaine group (phospho-GluR $\mathrm{R}_{1}$, $p<0.05$; phospho-NR $1, p<0.001$ ). When data were recalculated, statistical analysis indicated a significant difference in levels of phospho-GluR $\mathrm{R}_{1}$ and phospho-NR $\mathrm{NR}_{1}$ in the three

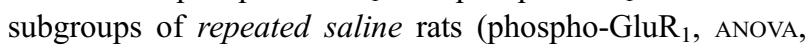
$F_{2,14}=28.49, \quad p<0.001 ; \quad$ phospho-NR,$\quad F_{2,13}=51.48$, $p<0.001)$. In rats in the repeated saline group, acute cocaine administration induced an increase in phosphoGluR $_{1}$ and phospho-NR $\mathrm{N}_{1}$ levels, compared to levels in the saline-treated rats, and this effect was already maximal at the $10 \mathrm{mg} / \mathrm{kg}$ dose (for both doses, phospho-GluR $1, p<0.01$ and phospho-NR $1, p<0.01$; Fig. $4 \mathrm{c}$ and d). In rats in the repeated cocaine group, significant differences were observed in the levels of phospho-GluR $\mathrm{R}_{1}$ and phospho-NR between different subgroups (phospho-GluR ${ }_{1}, F_{2,14}=30.20$, $p<0.001$; phospho-NR $\left.1, F_{2,12}=4.56, p<0.05\right)$ and, as observed with the DARPP-32 phosphorylation pattern, modifications in the levels of phosphorylated subunits were present only after the $20 \mathrm{mg} / \mathrm{kg}$ cocaine dose (phosphoGluR $_{1}, p<0.01$, phospho-NR $1, p<0.05$; Fig. 4c and d). These results indicated an increased threshold of PKAmediated phosphorylation of Thr34 DARPP-32, GluR 1 , and $\mathrm{NR}_{1}$ in the NAc of sensitized rats, possibly related to the steadily increased levels of phospho-Thr75 DARPP-32.

\section{Experiment A-3 - Acute mGluR5 inhibition in cocaine- sensitized rats: behavioral effects and modifications in levels of phosphorylation of DARPP-32, GluR 1 and $\mathrm{NR}_{1}$ subunits}

In order to investigate whether the expression of a sensitized behavioral response to cocaine and the steady increase in phospho-Thr75 DARPP-32 levels in sensitized rats was related to sustained mGluR5 activity, a selective antagonist of mGluR5s, MPEP, was acutely administered to control and cocaine-sensitized rats. When MPEP (5 mg/kg, i.p.) was administered, 10 days after the assessment of sensitization (Table 1), it did not modify the motility of rats in the repeated saline and repeated cocaine groups. Moreover, when cocaine $(10 \mathrm{mg} / \mathrm{kg}$, i.p.) was administered after MPEP, the expression of stereotypies and the increase in rearings were prevented in cocaine-sensitized, but not in control, rats (Table 2). Data were analyzed by ANOvA (stereotypy score: $F_{6,43}=11.89, \quad p<0.001$; rearing number: $F_{6,43}=3.88$, $p<0.01$; horizontal locomotor activity: $\mathrm{F}_{6,43}=3.73$, $p<0.01$ ), followed by Dunnett's test using the repeated saline rats receiving saline as the control group. This analysis confirmed the sensitized response to cocaine in the repeated cocaine group and showed an increase in stereotypies and motor activity in the repeated saline group (Table 2). Subsequently, the data relative to the repeated saline group and the repeated cocaine group were analyzed separately by ANOVA followed by Dunnett's test, using the respective saline + cocaine-treated group as the reference group. This analysis confirmed that MPEP administration before cocaine injection prevented the increase in stereotypies and rearings in cocaine-sensitized rats (stereotypy score $p<0.05$; rearing number $p<0.01)$.

The pattern of DARPP-32 phosphorylation and the levels of phospho-GluR $\mathrm{R}_{1}$, and phospho-NR $\mathrm{NR}_{1}$ were examined in the $\mathrm{mPFC}$ and NAc of rats in the repeated saline and repeated cocaine groups acutely administered saline $(1 \mathrm{~mL} / \mathrm{kg}$, i.p.), or the mGluR5 antagonist, MPEP $(5 \mathrm{mg} / \mathrm{kg}$, i.p.). In the mPFC, phospho-Thr75 DARPP-32 levels differed between groups $\left(F_{3,18}=22.53, p<0.001\right)$. Bonferroni's post hoc analysis demonstrated that in the repeated saline group phospho-Thr75 DARPP-32 levels decreased after MPEP administration (repeated saline $+\mathrm{MPEP}=80.4 \pm 4.8 \%$ of repeated saline, $p<0.01)$. Moreover, in the repeated cocaine group, phospho-Thr75 DARPP-32 levels decreased 
Table 2 Behavioral responses to cocaine challenge after saline or MPEP administration

\begin{tabular}{lllllc}
\hline \multirow{2}{*}{ Group } & Treatment & $n$ & $\begin{array}{l}\text { Stereotypy } \\
\text { score }\end{array}$ & $\begin{array}{l}\text { Rearing } \\
\text { number }\end{array}$ & $\begin{array}{l}\text { Horizontal } \\
\text { locomotor } \\
\text { activity }\end{array}$ \\
\hline Repeated saline & Saline + saline & 5 & $0.5 \pm 0.1$ & $19.6 \pm 5.0$ & $61.8 \pm 22.81$ \\
& Saline + cocaine & 7 & $2.4 \pm 0.5^{\S}$ & $179.6 \pm 54.7^{\S}$ & $423.4 \pm 117.7^{\S}$ \\
& MPEP + cocaine & 6 & $1.8 \pm 0.4$ & $110.0 \pm 46.5$ & $209.6 \pm 40.5$ \\
& MPEP + saline & 4 & $0.6 \pm 0.2$ & $21.0 \pm 5.3$ & $56.5 \pm 17.6^{*}$ \\
Repeated cocaine & Saline + cocaine & 8 & $4.1 \pm 0.3^{\S \S}$ & $172.0 \pm 18.3^{\S}$ & $374.9 \pm 67.4$ \\
& MPEP + cocaine & 8 & $2.6 \pm 0.5^{\S \S *}$ & $78.9 \pm 26.5^{\star *}$ & $358.8 \pm 125.5$ \\
& MPEP + saline & 5 & $0.4 \pm 0.2^{\star \star}$ & $25.6 \pm 10.9^{\star \star}$ & $39.4 \pm 20.4$ \\
\hline
\end{tabular}

${ }^{\S} p<0.05,{ }^{\S} p<0.01$ compared to the Repeated saline - Saline + saline group; ${ }^{*} p<0.05$, ${ }^{* *} p<0.01$ compared to the respective Saline + cocaine groups (ANOvA followed by Dunnett's test). after MPEP administration to levels similar to those observed in the repeated saline group (repeated cocaine $=$ $124.8 \pm 5.7 \%$ and repeated cocaine $+\mathrm{MPEP}=105.6 \pm$ $2.7 \%$ of repeated saline, repeated cocaine vs. repeated cocaine + MPEP $p<0.01)$. Phospho-Thr34 DARPP-32 levels differed between groups $\left(F_{3,19}=7.84, p<0.01\right)$, and post hoc analysis demonstrated that $1 \mathrm{~h}$ after MPEP administration, phospho-Thr34 DARPP-32 levels had increased in repeated cocaine rats to levels similar to those observed in repeated saline rats (repeated saline $+\mathrm{MPEP}=120.2 \pm$ $11.8 \%$, repeated cocaine $=80.4 \pm 2.5 \%$, repeated cocai$n e+\mathrm{MPEP}=113.8 \pm 3.6 \%$ of repeated saline; repeated cocaine vs. repeated cocaine $+\mathrm{MPEP}=p<0.01)$. Levels of phospho-GluR $R_{1}$ and phospho-NR $\mathrm{NR}_{1}$ subunits differed between groups (phospho-GluR $1, F_{3,18}=26.52, p<0.001$; phospho-NR $\left.1, F_{3,19}=9.13, p<0.001\right)$. In particular, post hoc analysis demonstrated that phospho-GluR $\mathrm{R}_{1}$ levels had increased in repeated saline rats after MPEP administration $(133.3 \pm 6.6 \%$ of saline-treated rats in the repeated saline group, $p<0.001)$, and a similar modification was observed in phospho-NR $\mathrm{NR}_{1}$ levels $(124.6 \pm 8.5 \%$ of saline-treated rats, $p<0.05)$. In rats of the repeated cocaine group, levels of phospho-GluR $\mathrm{R}_{1}$ subunit increased after MPEP administration $(108.2 \pm 4.0 \%$ vs. $83.4 \pm 3.5 \%$ in saline-treated repeated cocaine rats, $p<0.001$ ), and they were similar to repeated saline group levels. Similarly, phospho-NR 1 levels increased in rats of the repeated cocaine group after MPEP administration to levels similar to those observed in the repeated saline group $(113.0 \pm 8.1 \%$ vs. $81.8 \pm 3.0 \%$ in saline-treated repeated cocaine rats, $p<0.01)$.

In the NAc, phospho-Thr75 DARPP-32 levels differed between groups $\left(F_{3,19}=18.11, p<0.001\right)$ and post hoc Bonferroni's test demonstrated that levels were unmodified after MPEP administration in the repeated saline group; phospho-Thr75 DARPP-32 levels were lower in the repeated cocaine group after MPEP administration than in repeated cocaine rats receiving saline $(p<0.001)$, and similar to levels in repeated saline rats $(99.2 \pm 3.5 \%$ of repeated saline group; Fig. 5a). Phospho-Thr34 DARPP-32 levels were different between groups $\left(F_{3,19}=8.04, p<0.01\right)$ and post hoc analysis demonstrated that they were unmodified after MPEP administration in repeated saline rats (Fig. 5b). In repeated cocaine rats, phospho-Thr34 DARPP-32 levels increased $1 \mathrm{~h}$ after MPEP administration $(p<0.01)$ and were similar to levels in repeated saline rats $(99.8 \pm 6.45 \%$ of repeated saline group; Fig. 5b).

Levels of phospho-GluR $R_{1}$ and phospho-NR $\mathrm{NR}_{1}$ subunits in the NAc differed between groups (phospho-GluR $R_{1}$, $F_{3,19}=35.06, \quad p<0.001 ; \quad$ phospho-NR $1, \quad F_{3,19}=11.86$, $p<0.001)$. Post hoc analysis demonstrated that in repeated saline rats, phospho-GluR 1 levels increased after MPEP administration $(p<0.001)$, while phospho-NR $\mathrm{NR}_{1}$ levels were unmodified (Fig. $5 \mathrm{c}$ and d). On the other hand, in repeated cocaine rats, levels of phospho-GluR $\mathrm{R}_{1}$ and phospho-NR subunits increased after MPEP administration $(p<0.001$ for both phospho-GluR 1 and phospho-NR ; $_{1}$ Fig. $5 \mathrm{c}$ and d). These results showed that the acute administration of an mGluR5 antagonist reduced the expression of a sensitized response to a cocaine challenge and restored a pattern of DARPP-32, GluR 1 , and $\mathrm{NR}_{1}$ phosphorylation similar to that observed in control rats.

\section{Experiment B - Assessment of behavioral sensitization to cocaine and of the neurochemical modifications associated to repeated cocaine exposure after a long-term washout}

This experiment was aimed at verifying whether the observed neurochemical modifications associated to the expression of cocaine sensitization after a short-term washout were temporally related to behavioral sensitization. Thus, behavioral sensitization and the neurochemical variables studied after a short-term washout were determined after a 3-month washout in rats that had fully expressed behavioral sensitization after a 10-day washout.

In order to assess the development of sensitization, rats exposed to the sensitization protocol and rats from the salinetreated group were challenged with cocaine after a 10-day washout. As expected, cocaine-treated rats exhibited a sensitized response to the challenge (Table 1). However, when a subgroup of sensitized rats was challenged again 


\section{NAc}
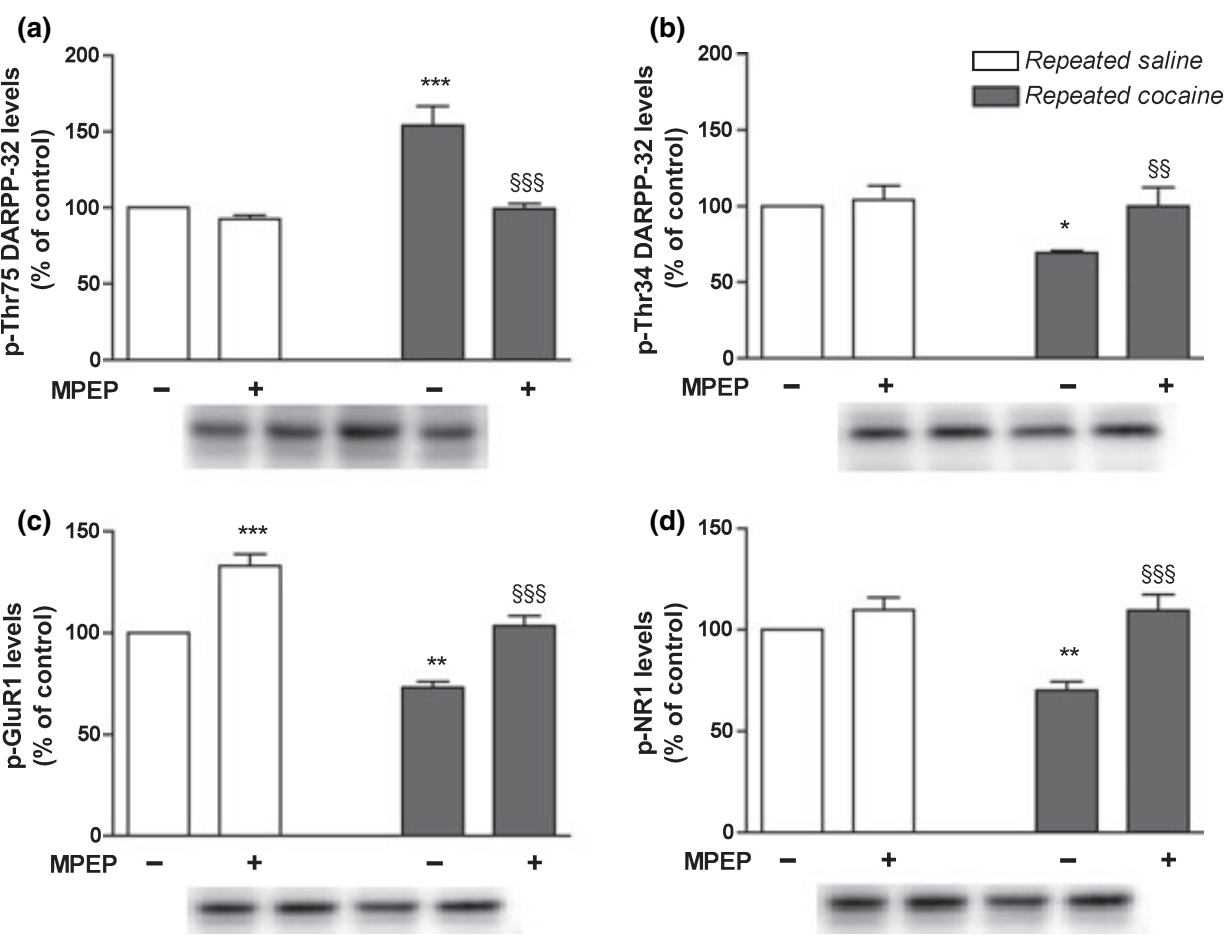

Fig. 5 Levels of phospho-Thr75 and phospho-Thr34 DARPP-32, phospho-GluR $\mathrm{R}_{1}$, and phospho-NR 1 in the NAc of control (repeated saline) and cocaine-sensitized (repeated cocaine) rats, after a saline $(1 \mathrm{~mL} / \mathrm{kg})$ or MPEP $(5 \mathrm{mg} / \mathrm{kg}$, i.p.) administration after a 10-day washout. Levels of phospho-Thr75 DARPP-32 (a), phospho-Thr34 DARPP-32 (b), phospho-GluR 1 (c), and phospho-NR $\mathrm{N}_{1}$ (d) are

3 months later (repeated cocaine ${ }_{3 m}$ group), the horizontal locomotion score was higher than that in the repeated saline $_{3 m}$ group ( $p<0.05$, unpaired $t$-test), but the stereotypy scores and number of rearings were similar to those of repeated saline ${ }_{3 m}$ group (Table 1). Thus, behavioral sensitization was still present after a 3-month washout, but it was less strongly expressed than after a short-term washout. Western blot analysis was then carried out in rats in the repeated saline $_{3 m}$ and repeated cocaine ${ }_{3 m}$ groups, exposed to saline or cocaine treatment before sacrifice.

\section{Western blot analysis in the mPFC: DARPP-32 phosphorylation pattern}

In the mPFC, a significant difference in phospho-Thr75 DARPP-32 levels was observed between groups $\left(F_{3,19}=10.96, p<0.001\right)$. Post hoc analysis demonstrated that in saline-treated rats, phospho-Thr75 DARPP-32 levels were similar in the two groups. After cocaine challenge, phospho-Thr75 DARPP-32 levels decreased in the repeated saline $_{3 m}$ and repeated cocaine $3 m$ groups, compared to their respective saline-treated groups $(p<0.01$ and $p<0.05$, respectively; Table 3). Phospho-Thr34 DARPP-32 levels differed significantly between groups $\left(F_{3,17}=13.46\right.$;

expressed as mean \pm SEM percentage of values in the saline-treated control group. Representative immunoblots are presented in the lower panels. ${ }^{\star \star *} p<0.001,{ }^{\star *} p<0.01,{ }^{\star} p<0.05$ versus the saline-treated repeated saline group; ${ }^{\S \S} p<0.001,{ }^{\S \S} p<0.01$ versus the salinetreated repeated cocaine group (Bonferroni's test).

$p<0.001$ ); they were similar in saline-treated rats and after cocaine administration they were significantly increased in the repeated saline $_{3 m}$ and repeated cocaine $3 m$ groups, compared to their respective saline-treated groups $(p<0.001$ and $p<0.05$, respectively; Table 3$)$.

\section{Levels of GluR $R_{1}$ and $\mathrm{NR}_{1}$ subunits and of their phosphorylated forms}

Analysis of the levels of $\mathrm{GluR}_{1}$ and $\mathrm{NR}_{1}$ subunits was not performed in the mPFC, as the expression of both subunits was unchanged in this region in control and sensitized rats after a 10-day washout. Phosphorylation levels of $\mathrm{GluR}_{1}$ and $\mathrm{NR}_{1}$ were examined in rats in the repeated saline ${ }_{3 m}$ and repeated cocaine $_{3 m}$ groups exposed to saline or cocaine administration. The levels of both phosphorylated subunits differed significantly between groups (phospho-GluR ${ }_{1}$, $F_{3,19}=8.74, p<0.01 ;$ phospho-NR ${ }_{1}, F_{3,16}=15.92, p<$ 0.001; Table 3). Post hoc analysis demonstrated that in saline-treated rats, levels of phospho-GluR ${ }_{1}$ and phospho$\mathrm{NR}_{1}$ were similar in the two groups. After cocaine challenge, levels of phospho-GluR $R_{1}$ significantly increased in both the repeated saline ${ }_{3 m}$ and repeated cocaine ${ }_{3 m}$ groups compared to their respective saline-treated groups $(p<0.01$ for the 
Table 3 Neurochemical modifications associated to repeated cocaine exposure after a 3-month washout

\begin{tabular}{|c|c|c|c|c|}
\hline & $\begin{array}{l}\text { Repeated } \\
\text { saline }_{3 m}\end{array}$ & $\begin{array}{l}\text { Repeated } \\
\text { saline }_{3 m}+ \\
\text { cocaine }\end{array}$ & $\begin{array}{l}\text { Repeated } \\
\text { cocaine }_{3 m}\end{array}$ & $\begin{array}{l}\text { Repeated } \\
\text { cocaine }_{3 m}+ \\
\text { cocaine }\end{array}$ \\
\hline \multicolumn{5}{|l|}{$m P F C$} \\
\hline Thr75-DARPP32 & 100 & $70.6 \pm 5.4^{\star \star}$ & $107.0 \pm 7.1$ & $83.8 \pm 4.3^{\S}$ \\
\hline Thr34-DARPP32 & 100 & $134.0 \pm 3.2^{\star \star *}$ & $98.25 \pm 4.8$ & $119.8 \pm 6.7^{*}$ \\
\hline pGluR $_{1}$ & 100 & $135.0 \pm 7.7^{\star *}$ & $103.0 \pm 5.3$ & $126.2 \pm 7.0^{\S}$ \\
\hline $\mathrm{pNR}_{1}$ & 100 & $130.8 \pm 4.9^{* \star *}$ & $106.8 \pm 3.0$ & $124.8 \pm 4.0^{\S \S}$ \\
\hline Cdk5 & 100 & ND & $105.5 \pm 3.5$ & ND \\
\hline \multicolumn{5}{|l|}{$N A C$} \\
\hline Thr75-DARPP32 & 100 & $51.8 \pm 4.6^{\star \star \star}$ & $109.0 \pm 8.0$ & $83.0 \pm 6.9^{\S}$ \\
\hline Thr34-DARPP32 & 100 & $184.0 \pm 12.1^{\star \star \star}$ & $100.4 \pm 8.1$ & $125.2 \pm 10.9$ \\
\hline GluR $_{1}$ & 100 & ND & $94.4 \pm 3.5$ & ND \\
\hline $\mathrm{NR}_{1}$ & 100 & ND & $82.2 \pm 9.7$ & ND \\
\hline pGluR $_{1}$ & 100 & $185.8 \pm 15.2^{\star \star \star}$ & $108.6 \pm 2.1$ & $144.6 \pm 10.3^{\S}$ \\
\hline $\mathrm{pNR}_{1}$ & 100 & $174.0 \pm 14.0^{* \star *}$ & $103.4 \pm 4.1$ & $139.6 \pm 5.6^{\S}$ \\
\hline Cdk5 & 100 & ND & $106+6.0$ & ND \\
\hline
\end{tabular}

Levels of phospho-Thr75 and phospho-Thr34 DARPP-32, GluR, NR $_{1}$, phospho-GluR 1 , phospho$\mathrm{NR}_{1}$ and Cdk5 in the mPFC and NAc of control (repeated saline ${ }_{3 m}$ ) and cocaine-sensitized (repeated cocaine $\left.{ }_{3 m}\right)$ rats, after a saline $(0.1 \mathrm{~mL} / \mathrm{kg})$ or cocaine $(10 \mathrm{mg} / \mathrm{kg}$, i.p.) challenge, administered after a 3-month washout. Levels of phospho-Thr75 and phospho-Thr34 DARPP-32, GluR $_{1}, \mathrm{NR}_{1}$, phospho-GluR 1 , phospho-NR ${ }_{1}$, and Cdk5 are expressed as mean $\pm \mathrm{SEM}$ percentage of levels in the saline-treated control (repeated saline $3 m$ ) rats. ${ }^{\star * *} p<0.001,{ }^{\star *} p<0.001,{ }^{*} p<0.05$ versus the saline-treated repeated saline ${ }_{3 m}$ group; ${ }^{\S} p<0.001,{ }^{\S} p<0.05$ versus the saline-treated repeated cocaine ${ }_{3 m}$ group.

repeated saline $_{3 m}$ group and $p<0.05$ for the repeated cocaine $_{3 m}$ group; Table 3). Phospho-NR 1 levels were also significantly increased in the repeated saline $_{3 m}$ group and in the repeated cocaine $_{3 m}$ group compared to their respective saline-treated groups $(p<0.001$ for the repeated saline $3 m$ group and $p<0.01$ for the repeated cocaine $3 m$ group; Table 3).

\section{Cdk5 levels}

After a 3-month washout, Cdk5 levels were similar in the repeated saline $_{3 m}$ and repeated cocaine ${ }_{3 m}$ groups (Table 3 ).

These results demonstrated that in the mPFC of sensitized rats, after a 3-month washout, Thr75 DARPP-32, Thr34 DARPP-32, GluR ${ }_{1}$, and $\mathrm{NR}_{1}$ phosphorylation levels, as well as Cdk5 levels, were similar to those of age-matched control rats.

\section{Western blot analysis in the NAc: DARPP-32 phosphorylation pattern}

In the NAc, phospho-Thr75 DARPP-32 levels differed significantly between groups $\left(F_{3,19}=19.21, p<0.001\right)$. Post hoc analysis demonstrated that in saline-treated rats, phospho-Thr75 DARPP-32 levels were similar in the two groups. After acute cocaine administration, phospho-Thr75 DARPP-32 levels decreased in the repeated saline $_{3 m}$ and repeated cocaine $_{3 m}$ groups, compared to levels in their respective saline-treated groups $(p<0.001$ and $p<0.05$, respectively; Table 3). Phospho-Thr34 DARPP-32 levels were also significantly different between groups $\left(F_{3,19}=18.77, p<0.001\right)$. In saline-treated rats, levels in the repeated cocaine $3 m$ group were similar to levels in the repeated saline ${ }_{3 m}$ group. After acute cocaine, phospho-Thr34 DARPP-32 levels increased in the repeated saline $_{3 m}$ group compared to the saline-treated repeated saline $_{3 m}$ rats $(p<0.001)$, while in the repeated cocaine $_{3 m}$ group they were similar to the levels in saline-treated repeated cocaine ${ }_{3 m}$ rats (Table 3).

\section{Levels of GluR $R_{1}$ and $\mathrm{NR}_{1}$ subunits and of their phosphorylated forms}

Analysis of the levels of $\mathrm{GluR}_{1}$ and $\mathrm{NR}_{1}$ subunits indicated that the expression of both subunits was similar in rats in the repeated saline $_{3 m}$ and in the repeated cocaine $3 m$ groups (Table 3). Analysis of phosphorylation levels of $\mathrm{GluR}_{1}$ and $\mathrm{NR}_{1}$ subunits in rats in the repeated saline ${ }_{3 m}$ and repeated cocaine $_{3 m}$ groups indicated a significant difference between groups (phospho-GluR ${ }_{1}, F_{3,19}=17.90, p<0.001$; phospho$\left.\mathrm{NR}_{1}, \quad F_{3,19}=19.90, p<0.001\right)$. In saline-treated rats, phospho-GluR 1 and phospho-NR $\mathrm{NR}_{1}$ levels were similar in the repeated saline $_{3 m}$ and repeated cocaine $_{3 m}$ groups (Table 3). After acute cocaine challenge, phospho-GluR $\mathrm{R}_{1}$ and phospho-NR $\mathrm{N}_{1}$ levels increased in both the repeated saline $_{3 m}$ and repeated cocaine ${ }_{3 m}$ groups, compared to the levels in their respective saline-treated groups (phosphoGluR $_{1}$ and phospho-NR $1, p<0.001$ repeated saline ${ }_{3 m}$ and $p<0.05$ for the repeated cocaine $3 m$ group; Table 3). 


\section{Cdk5 levels}

After a 3-month washout, Cdk5 levels in the NAc were similar in the repeated saline $_{3 m}$ and repeated cocaine $_{3 m}$ groups (Table 3).

The results of experiment B indicated that, in the NAc of rats fully sensitized after a short-term washout, neurochemical variables were in part similar to control group values after a 3-month washout: i.e. phospho-Thr75 DARPP-32, phospho-Thr34 DARPP-32, GluR $1, \mathrm{NR}_{1}$, phospho-GluR 1 , phospho-NR $\mathrm{NR}_{1}$, and $\mathrm{Cdk} 5$ levels in saline-treated rats were similar in the repeated cocaine $_{3 m}$ and repeated saline $3 m$ groups. After cocaine challenge, phospho-Thr75 DARPP-32, phospho-GluR ${ }_{1}$, and phospho-NR $\mathrm{NR}_{1}$ levels changed in the same directions as in the repeated saline $_{3 m}$ group, although to a lesser extent (repeated cocaine ${ }_{3 m}$ vs. repeated saline $3 m$ $p<0.01$ for phospho-Thr75 DARPP-32, $p<0.05$ for both phospho-GluR $_{1}$ and phospho-NR N $_{1}$, while phospho-Thr34 DARPP-32 levels were unmodified.

\section{Expression of $\Delta \mathrm{FosB}$ after a short- and long-term washout period}

In order to study whether modifications in $\Delta \mathrm{FosB}$ levels followed a similar temporal pattern of the behavioral expression of sensitization and of the neurochemical modifications in the DARPP-32/PKA pathway, the expression of $\Delta$ FosB was examined in the NAc of rats in the repeated saline and repeated cocaine groups and in the repeated saline ${ }_{3 m}$ and repeated cocaine $3 m$ groups, i.e. in rats killed after a 10-day or 3 -month washout. Rats from the two experiments were killed at the same time and tissues were processed simultaneously. ANOVA analysis of the data revealed a significant difference between the groups $\left(F_{3,19}=15.60, p<0.001\right)$. Post hoc analysis indicated that the levels of $\Delta \mathrm{FosB}$ expression were increased in the repeated cocaine group compared to the repeated saline group ( $p<0.05$, Fig. $6 \mathrm{a}, \mathrm{b}$, and $\mathrm{c}$ ), while $\Delta$ FosB expression was similar in the repeated cocaine $3 m$ and repeated saline $_{3 m}$ groups (Fig. 6a, d, and e). Moreover, $\Delta$ FosB levels were higher in the repeated saline $3 m$ group compared to those in the repeated saline group $(p<0.01$, Fig. 6a, b, and d). The anti-FosB antibody recognizes all forms of FosB, but we assume that in sensitized rats the increased immunostain represents $\triangle \mathrm{FosB}$ as previous studies have shown that $\Delta \mathrm{FosB}$, but not FosB or other Fos-related antigens, is steadily expressed after repeated cocaine treatment (Nestler 2001). However, we cannot be certain of the identity of the immunoreactive FosB signal observed in the NAc of repeated saline $_{3 m}$ rats.

\section{Discussion}

The present study shows that a protocol of repeated cocaine administration, that induced in rats a condition of clear-cut behavioral sensitization, modified the pattern of DARPP-32 phosphorylation and the levels of $\mathrm{Cdk} 5, \mathrm{GluR}_{1}$, phospho-
GluR $_{1}, \mathrm{NR}_{1}$, and phospho-NR $\mathrm{NR}_{1}$ in both the mPFC and NAc after a short-term washout period (10 days). Moreover, after a long-term washout ( 3 months) the expression of behavioral sensitization was still present, although attenuated, and the modifications in the same neurochemical markers were only conserved to a certain extent. The present findings confirmed previous data obtained in rats with a different sensitization protocol (40 mg/kg on alternate days for 14 days) (Scheggi et al. 2004a,b).

Acute cocaine administration in sensitized rats elicited the expected sensitized behavioral response, yet it did not affect the pattern of modifications in DARPP-32, GluR 1 , and $\mathrm{NR}_{1}$ phosphorylation. This lack of effect was observed selectively in the NAc, whereas DARPP-32 responsiveness to a $10 \mathrm{mg} /$ $\mathrm{kg}$ cocaine challenge was preserved in the mPFC. Only a dose of $20 \mathrm{mg} / \mathrm{kg}$ of cocaine, double the efficacious dose in control rats, was able to decrease the levels of phosphoThr75 DARPP-32 and increase those of phospho-Thr34 DARPP-32 in the NAc of sensitized animals. Thus, the increase in dopamine levels elicited by a $10 \mathrm{mg} / \mathrm{kg}$ cocaine administration lacked the ability to increase PKA activity and subsequently phospho-Thr34 DARPP-32 levels in the NAc of cocaine-sensitized rats. These results suggest that the pattern of DARPP-32 phosphorylation in the NAc of cocaine-sensitized rats, characterized by high phosphoThr75 DARPP-32 levels, may exert an inhibitory effect on PKA. Moreover, this blunted responsiveness of the PKA/ DARPP-32 pathway to acute cocaine administration could be a neurochemical marker of the condition of cocaine sensitization. We then attempted to elucidate the mechanism that sustained the functional prevalence of phospho-Thr75 DARPP-32 in cocaine-sensitized rats. Metabotropic glutamate receptors have been reported to be involved in many of the acute and chronic effects of cocaine exposure. Thus, cocaine does not affect motility and does not induce self-administration in mGluR5 knock out mice (Chiamulera et al. 2001), and mGluR 1 and mGluR2/3 are involved in the increased release of synaptic glutamate in the NAc and in the reinstatement of cocaine seeking in rats extinguished from cocaine self-administration (Kalivas et al. 2003). Metabotropic glutamate receptors also play a direct role in DARPP32 phosphorylation, in particular, in neostriatal slices, the activation of $\mathrm{Cdk} 5$ and phosphorylation of Thr75 DARPP-32 have been associated with the activation of type I mGluR (Liu et al. 2001). Moreover, we have previously demonstrated that a transient increase in phospho-Thr75 DARPP-32 levels, induced by palatable food consumption, is prevented by the administration of MPEP, an antagonist of the mGluR $_{5}$ subtype of type I mGluRs (Rauggi et al. 2005). Acute MPEP administration to sensitized rats reinstated the levels of phosphorylation of Thr75- and Thr34-DARPP-32 and of GluR 1 and $\mathrm{NR}_{1}$ subunits to the values of control animals. In fact, MPEP significantly reduced the levels of phosphoThr75 DARPP-32 and increased those of phospho-Thr34 


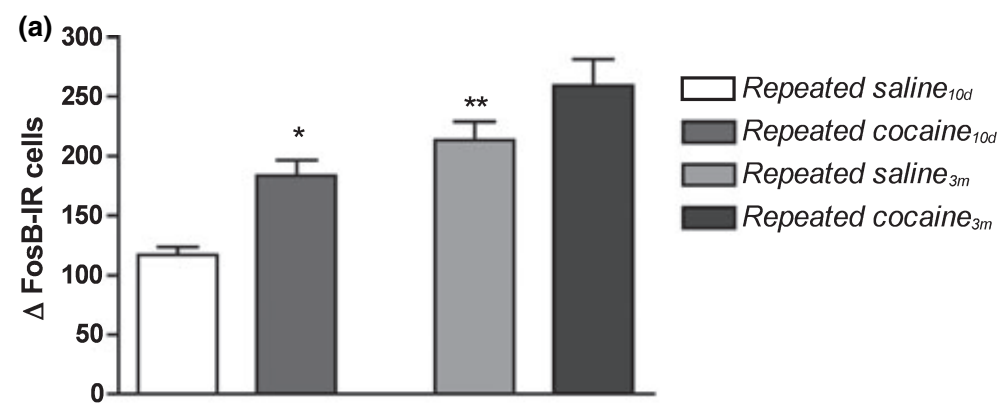

(b)

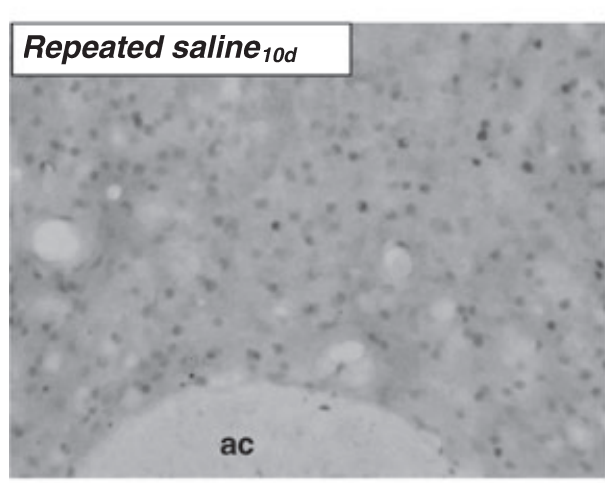

(d)

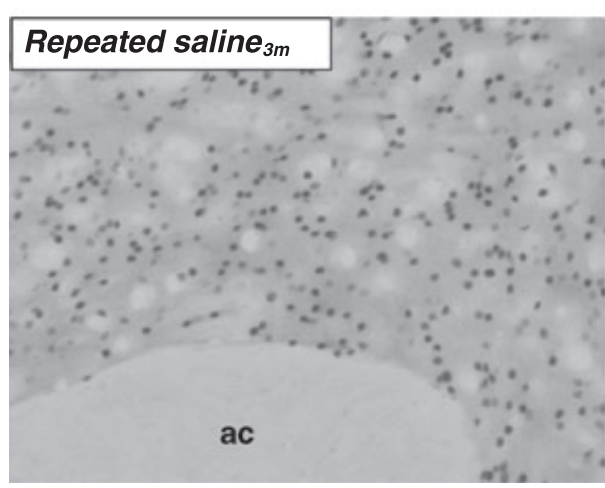

Fig. 6 Expression of $\Delta$ FosB in control and cocaine-sensitized rats after a 10-day or 3-month washout. Cumulative data (number of $\Delta$ FosB-like-immunoreactive cells) are shown in graphical format (a). Photomicrographs of $\triangle$ FosB-like-immunoreactive cells in the NAc of

DARPP-32, phospho-GluR $R_{1}$, and phospho-NR $\mathrm{N}_{1}$ subunits in the $\mathrm{mPFC}$ and NAc of sensitized rats. These results suggest that in cocaine-sensitized rats a tonic stimulation of mGluR $5 \mathrm{~s}$ maintains DARPP-32 in the prevalent functional conformation of PKA inhibitor. In control rats, MPEP administration had modest effects in the NAc, as only phospho-GluR $\mathrm{R}_{1}$ levels were increased; whereas, in the $\mathrm{mPFC}$ it decreased the levels of phospho-Thr75 DARPP-32 and increased those of phospho-Thr34 DARPP-32, phospho-GluR ${ }_{1}$, and phospho$\mathrm{NR}_{1}$. These results suggest that glutamatergic transmission in the $\mathrm{mPFC}$ is characterized by a tonic stimulation of mGlu5 receptors. From a behavioral point of view, acute MPEP administration did not significantly modify the motor activity
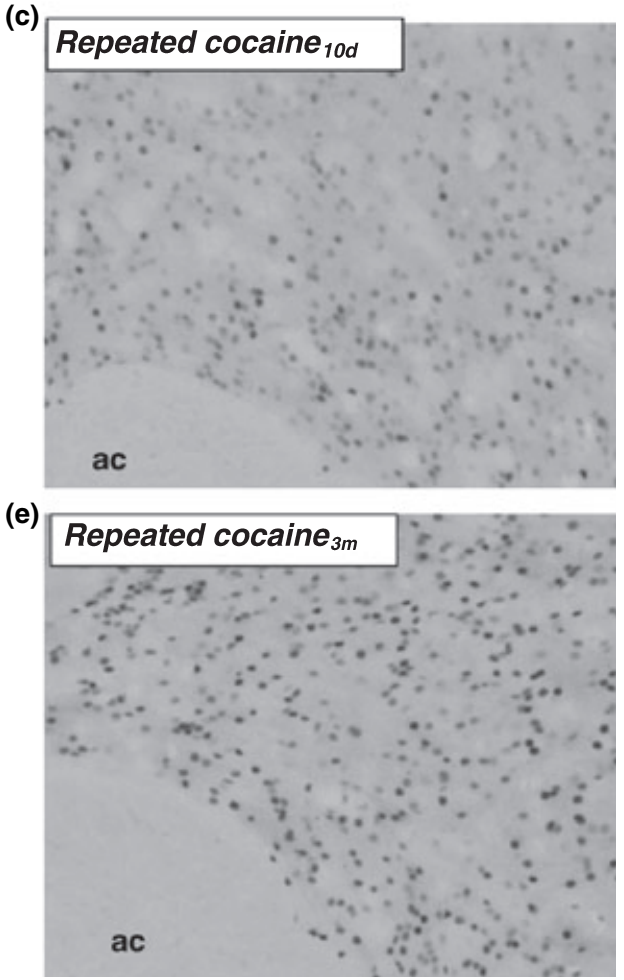

control and cocaine-sensitized rats after a 10-day [repeated saline ${ }_{10 \mathrm{~d}}$ (b); repeated cocaine ${ }_{10 d}$ (c)] or 3-month washout [repeated saline $3 m$ (d), repeated cocaine $3 m$ (e)]. Original magnification: $20 \times .{ }^{\star \star} p<0.01$, ${ }^{\star} p<0.05$ versus the repeated saline ${ }_{10 d}$ group (Bonferroni's test).

of control and sensitized rats, but it reduced the sensitized response to a cocaine challenge, thus suggesting that the modified functional balance between the PKA inhibitory and amplificatory forms of DARPP-32 is related to the condition and expression of a cocaine-sensitized state. These data support the hypothesis that increased phospho-Thr75 DARPP-32 levels are strictly linked to the condition of cocaine sensitization, and they are reminiscent of those obtained in the mutant mice that cannot phosphorylate DARPP-32 at Thr75 and do not develop cocaine sensitization (Zachariou et al. 2006).

After the long-term (3 month) washout, behavioral sensitization was less robustly expressed than after a 
short-term washout, as horizontal locomotor activity was still significantly increased while stereotypy scores were similar to those of control rats. Different results have been reported in terms of persistence of cocaine sensitization, from 1-2 months up to 6 months (Henry and White 1995; Hope et al. 2006). These results are probably related to differences in the sensitization treatment regimen chosen (repeated cocaine administration in the home-cage vs. novel-environment, variability in the dose of cocaine, number of administrations, and interval between administrations), but also in the different criteria selected for the assessment of sensitization (e.g. stereotypies vs. horizontal locomotor activity).

After the 3-month washout, the DARPP-32 phosphorylation pattern and GluR 1 , phospho-GluR $1, \mathrm{NR}_{1}$, phospho-NR $\mathrm{N}_{1}$, and $\mathrm{Cdk} 5$ levels were similar to those observed in agematched control rats, in both the mPFC and NAc. It should be noted that when basal phospho-Thr75 DARPP-32 levels reached control values, the levels of Cdk5 expression were decreased, in agreement with the proposed role of this kinase as a negative regulator of dopamine transmission in cocainesensitized animals (Taylor et al. 2007). The responsiveness of the DARPP-32 system to a cocaine challenge was still unmodified in the mPFC. In the NAc, the response to an acute cocaine challenge was only partially restored, compared to the response of age-matched control rats. In fact, phospho-Thr75 DARPP-32 levels decreased and phosphoGluR $_{1}$ and phospho-NR $\mathrm{NR}_{1}$ levels increased, although to a lesser extent than in control rats, while phospho-Thr34 DARPP-32 levels were unmodified. Finally, the levels of $\Delta$ FosB-like proteins in the NAc were similar to those in the age-matched control group. Moreover, the levels of $\Delta \mathrm{FosB}$ like immunoreactivity in the control animals of the 3-month washout experiment were higher than those in the control animals of the 10-day washout experiment. A progressive developmental increase in $\Delta$ FosB-like expression in the NAc during the post-weanling, periadolescent, and adult ages has been described in mice (Ehrlich et al. 2002); however, a thorough study of $\Delta$ FosB-like protein levels as a function of aging has not been performed. Thus, we do not know whether aging alone and/or other factors in our experimental conditions contributed to the observed induction of $\Delta \mathrm{FosB}$ like levels.

In conclusion, behavioral sensitization to cocaine is a long-lasting, yet transient phenomenon, at least in our experimental setting, and we have shown that the neurochemical modifications that characterize the condition of sensitization and the sensitized behavioral response to cocaine have a similar temporal pattern. In particular, after a 10-day washout, the behavioral expression of sensitization was accompanied by neurochemical modifications in the dopamine $\mathrm{D}_{1}$ receptor/DARPP-32/PKA pathway, and after a 3-month washout, both the behavioral expression and the neurochemical modifications were still present, but in an attenuated form. Thus, the present data support the correlation between the condition of cocaine-induced behavioral sensitization and a tonic increase in the levels of phosphoThr75 DARPP-32 that inhibits PKA activity. This increase was sustained by mGluR5 stimulation, leading to complex neuroadaptation processes that lasted as long as behavioral sensitization.

\section{Acknowledgments}

This study was supported by a research grant from SIGMA TAU Industrie Farmaceutiche Riunite S.p.A., Pomezia, Italy. The authors wish to thank Ms Colleen Pisaneschi for language editing of the manuscript.

\section{References}

Baker D. A., McFarland K., Lake R. W., Shen H., Tang X. C., Toda S. and Kalivas P. W. (2003) Neuroadaptations in cystine-glutamate exchange underlie cocaine relapse. Nature Neurosci. 6, 743-749.

Bibb J. A., Snyder G. L., Nishi A. et al. (1999) Phosphorylation of DARPP- 32 by Cdk 5 modulates dopamine signalling in neurons. Nature 402, 669-671.

Bibb J. A., Chen J., Taylor J. R. et al. (2001) Effects of chronic exposure to cocaine are regulated by the neuronal protein $\mathrm{Cdk} 5$. Nature $\mathbf{4 1 0}$, 376-379.

Chiamulera C., Epping-Jordan M. P., Zocchi A., Marcon C., Cottiny C., Tacconi S., Corsi M., Orzi F. and Conquet F. (2001) Reinforcing and locomotor stimulant effects of cocaine are absent in mGluR5 null mutant mice. Nature Neurosci. 4, 873-874.

Ehrlich M. E., Sommer J., Canas E. and Unterwald E. M. (2002) Periadolescent mice show enhanced DFosB upregulation in response to cocaine and amphetamine. J. Neurosci. 22, 9155-9159.

Gambarana C., Ghiglieri O., De Montis M. G. and Tagliamonte A. (1998) Under continuous dizocilpine infusion an N-methyl-Daspartate receptor independent form of cocaine sensitization develops in rats. Behav. Pharmacol. 9, 61-68.

Greengard P. (2001) The neurobiology of slow synaptic transmission. Science 294, 1024-1030.

Hemmings H. C. Jr, Greengard P., Tung H. Y. and Cohen P. (1984) DARPP-32, a dopamine regulated neuronal phosphoprotein, is a potent inhibitor of protein phosphatase-1. Nature 310, 503-505.

Henry D. J. and White F. J. (1995) The persistence of behavioral sensitization to cocaine parallels enhanced inhibition of nucleus accumbens neurons. J. Neurosci. 15, 6287-6299.

Hope B. T., Crombag H. S., Jedynak J. P. and Wise R. A. (2005) Neuroadaptations of total levels of adenylate cyclase, protein kinase A, tyrosine hydroxylase, cdk5 and neurofilaments in the nucleus accumbens and ventral tegmental area do not correlate with expression of sensitized or tolerant locomotor responses to cocaine. J. Neurochem. 92, 536-545.

Hope B. T., Simmons D. E., Mitchell T. B., Kreuter J. D. and Mattson B. J. (2006) Cocaine-induced locomotor activity and Fos expression in nucleus accumbens are sensitized for 6 months after repeated cocaine administration outside the home cage. Eur. J. Neurosci. 24, 867-875.

Kalivas P. W. (2004) Glutamate systems in cocaine addiction. Curr. Opin. Pharmacol. 4, 23-29.

Kalivas P. W., McFarland K., Bowers S., Szumlinski K., Xi Z. X. and Baker D. (2003) Glutamate transmission and addiction to cocaine. Ann. N. Y. Acad. Sci. 1003, 169-175. 
Karler R., Calder L. D., Chaudry I. A. and Turkanis S. A. (1989) Blockade of 'reverse tolerance' to cocaine and amphetamine by MK-801. Life Sci. 45, 599-606.

Kenny P. J. and Markou A. (2004) The ups and downs of addiction: role of metabotropic glutamate receptors. Trends Pharmacol. Sci. 25, $265-272$.

Liu F., Ma X. H., Ule J., Bibb J. A., Nishi A., DeMaggio A. J., Yan Z., Nairn A. C. and Greengard P. (2001) Regulation of cyclindependent kinase 5 and casein kinase 1 by metabotropic glutamate receptors. Proc. Natl Acad. Sci. USA 98, 11062-11068.

Lowry O. H., Rosebrough N. J., Farr A. L. and Randall R. J. (1951) Protein measurement with the Folin phenol reagent. J. Biol. Chem. 193, 265-275.

McClung C. A., Ulery P. G., Perrotti L. I., Zachariou V., Berton O. and Nestler E. J. (2004) FosB: a molecular switch for long-term adaptation. Mol. Brain Res. 132, 146-154.

Nakabeppu Y. and Nathans D. (1991) A naturally occurring truncated form of FosB that inhibits Fos/Jun transcriptional activity. Cell 64, 751-759.

Nestler E. J. (2001) Molecular basis of long term adaptations underlying addiction. Nat. Rev. Neurosci. 2, 119-128.

Nishi A., Bibb J. A., Snyder G. L., Higashi H., Nairn A. C. and Greengard P. (2000) Amplification of dopaminergic signaling by a positive feedback loop. Proc. Natl Acad. Sci. USA 97, 1284012845.

Ouimet C. C., Miller P. E., Hemmings H. C. Jr, Walaas S. I. and Greengard P. (1984) DARPP-32, a dopamine- and adenosine $3^{\prime}: 5^{\prime}$ monophosphate-regulated phosphoprotein enriched in dopamineinnervated brain regions. III. Immunocytochemical localization. J. Neurosci. 4, 111-124.

Paterson N. E., Semenova S., Gasparini F. and Markou A. (2003) The mGluR5 antagonist MPEP decreased nicotine self-administration in rats and mice. Psychopharmacology 167, 257-264.

Paxinos G. and Watson C. (1998) The Rat Brain in Stereotaxic Coordinates. Academic Press, New York.

Rauggi R., Scheggi S., Cassanelli A., De Montis M. G., Tagliamonte A. and Gambarana C. (2005) The mesolimbic dopaminergic response to novel palatable food consumption increases dopamine-D1 receptor-mediated signalling with complex modifications of the DARPP-32 phosphorylation pattern. J. Neurochem. 92, 867-877.

Scheggi S., Rauggi R., Gambarana C., Tagliamonte A. and De Montis M. G. (2004a) Dopamine and cyclic AMP-regulated phosphoprotein-32 phosphorylation pattern in cocaine and morphine-sensitized rats. J. Neurochem. 90, 792-799.

Scheggi S., Rauggi R., Nanni G., Tagliamonte A. and Gambarana C. (2004b) Repeated acetyl-L-carnitine administration increases phospho-Thr34 DARPP-32 levels and antagonizes cocaine-induced increase in Cdk5 and phospho-Thr75 DARPP-32 levels in rat striatum. Eur. J. Neurosci. 19, 1609-1620.

Shippenberg T. S., LeFevour A. and Thompson A. C. (1998) Sensitization to the conditioned rewarding effects of morphine and cocaine: differential effects of the kappa-opioid receptor agonist U69593. Eur. J. Pharmacol. 345, 27-34.

Svenningsson P., Tzavara E. T., Carruthers R., Rachleff I., Wattler S., Nehls M., McKinzie D. L., Fienberg A. A., Nomikos G. G. and Greengard P. (2003) Diverse psychotomimetics act through a common signaling pathway. Science 302, 1412-1415.

Taylor J. R., Lynch W. J., Sanchez H., Olausson P., Nestler E. J. and Bibb J. A. (2007) Inhibition of Cdk5 in the nucleus accumbens enhances the locomotor-activating and incentive-motivational effects of cocaine. Proc. Natl Acad. Sci. USA 104, 4147-4152.

Vanderschuren L. J. and Kalivas P. W. (2000) Alterations in dopaminergic and glutamatergic transmission in the induction and expression of behavioral sensitization: a critical review of preclinical studies. Psychopharmacology 151, 99-120.

Walaas S. I., Aswad D. W. and Greengard P. (1983) A dopamine- and cyclic AMP regulated phosphoprotein enriched in dopamineinnervated brain regions. Nature 301, 69-71.

Wolf M. E. and Jeziorski M. (1993) Coadministration of MK-801 with amphetamine, cocaine or morphine prevents rather than transiently masks the development of behavioral sensitization. Brain Res. 613, 291-294.

Zachariou V., Sgambato-Faure V., Sasaki T., Svenningsson P., Berton O., Fienberg A. A., Nairn A. C., Greengard P. and Nestler E. J. (2006) Phosphorylation of DARPP-32 at Threonine-34 is required for cocaine action. Neuropsychopharmacology 31, 555-562. 